JOURNAL OF THE

AMERICAN MATHEMATICAL SOCIETY

Volume 17, Number 3, Pages 557-593

S 0894-0347(04)00457-6

Article electronically published on April 16, 2004

\title{
A SINGULARITY REMOVAL THEOREM FOR YANG-MILLS FIELDS IN HIGHER DIMENSIONS
}

TERENCE TAO AND GANG TIAN

\section{INTRODUCTION}

The purpose of this paper is to investigate the small-energy behavior of weakly Yang-Mills fields in $\mathbf{R}^{n}$ for $n \geq 4$, and in particular to extend the singularity removal theorem of Uhlenbeck [7] to higher dimensions.

Fix $n \geq 4$, and let $\Omega$ be some bounded domain in $\mathbf{R}^{n}$; typically we shall restrict our attention to the cubes $\Omega=[-1,2]^{n}$ or $\Omega=[0,1]^{n}$.

Let $G$ be a fixed finite-dimensional compact Lie group; it will be convenient to consider $G$ as embedded in some large unitary group $U(N)$. Let $\mathfrak{g}$ be the Lie algebra of $G$. We define a connection on $\Omega$ to be a section $A$ of $T^{*} \Omega \otimes \mathfrak{g}$ (i.e., a $\mathfrak{g}$-valued 1-form) which is locally $L^{2}$. For any connection $A$ let

$$
F(A):=d A+A \wedge A
$$

denote the curvature of $A$. Since $A$ is locally $L^{2}, F(A)$ makes sense as a (g-valued 2 -form) distribution.

A gauge transformation is a sufficiently regulat 1 map $\sigma: \Omega \rightarrow G$. This group acts on connections by the formula

$$
\sigma(A):=\sigma \cdot A \cdot \sigma^{-1}-d \sigma \cdot \sigma^{-1} .
$$

We call $A$ and $\sigma(A)$ gauge equivalent.

Let $A$ be a connection on the cube $[-1,2]^{n}$. We say that $A$ is a smooth Yang-Mills connection on $[-1,2]^{n}$ if $A$ is smooth and solves the PDE

$$
d_{*} F(A)-*[A, * F(A)]=0,
$$

where $*$ denotes the Hodge operator and $d_{*}:=* d *$; one may verify that this condition is invariant under gauge transformations. Formally, Yang-Mills connections are critical points of the energy functional $\int|F(A)|^{2}$. Following [5], we say that $A$ is an admissible Yang-Mills connection if it is a smooth Yang-Mills connection outside a closed subset $S \subset[-1,2]^{n}$ of finite $(n-4)$-dimensional Hausdorff measure and $\int|F(A)|^{2}<\infty$. We will call $S$ the singular set of $S$.

Received by the editors September 27, 2002.

2000 Mathematics Subject Classification. Primary 53C07.

Key words and phrases. Yang-Mills fields, gauge transformation, monotonicity formula, Morrey spaces.

The first author is a Clay Prize Fellow and is supported by a grant from the Packard Foundation.

The second author is supported by an NSF grant and a Simons fund.

${ }^{1}$ As a bare minimum, one should have $\sigma$ in the Sobolev space $W_{l o c}^{1,2}$ and also in $L^{\infty}$, in order for $\sigma(A)$ to be locally in $L^{2}$. In practice we shall have significantly more regularity than this. 
It follows from analysis in [5] that weak limits of smooth Yang-Mills connections with curvature uniformly $L^{2}$-bounded are admissible Yang-Mills connections.

Following [5], we call an admissible Yang-Mills connection A stationary if for any vector field $X=X^{i} \frac{\partial}{\partial x_{i}}$ with compact support in $(-1,2)^{n}$, we have

$$
\int_{[-1,2]^{n}}\left(\left|F_{A}\right|^{2} \operatorname{div}(X)-4 F_{\alpha \beta} F_{\alpha \gamma} \frac{\partial X^{\beta}}{\partial x_{\gamma}}\right)=0,
$$

where $F_{A}=F_{\alpha \beta} d x_{\alpha} \wedge d x_{\beta}$ and we use the usual summation conventions. It follows from a monotonicity formula of Price [2] that $r^{4-n} \int_{B(x, r)}\left|F_{A}\right|^{2}$ is monotone nondecreasing for any stationary Yang-Mills connection $A$ (cf. [5]).

The main result of this paper is the following singularity removal theorem for small energy stationary admissible Yang-Mills connections:

Theorem 1.1. Let $A$ be a stationary admissible Yang-Mills connection on $[-1,2]^{n}$ with singular set $S$ which obeys the smallness condition

$$
\int_{[-1,2]^{n}}|F(A)|^{2} \leq \varepsilon .
$$

Then, if $0<\varepsilon \ll 1$ is sufficiently small (depending only on $n, G, N$ ), there is a gauge transformation $\sigma$ on $[0,1]^{n} \backslash S$ such that $\sigma(A)$ extends to a smooth connection over all of $[0,1]^{n}$. Indeed, we have the uniform bounds

$$
\left|\nabla^{j} \sigma(A)(x)\right| \leq C_{j} \varepsilon
$$

for all $x \in[0,1]^{n}$ and $j=0,1, \ldots$.

When $n=4$, the stationary property is automatic for admissible Yang-Mills connections. Hence, the above theorem generalizes the results of Uhlenbeck ([6], 7]), who proved the above removable singularity theorem in four dimensions. A proof of this theorem was given in [5] under the assumption of existence of a good gauge. The construction of this good gauge was unknown then even in the case that $S$ is a smooth submanifold 3 The main technical part of this paper is to fill in this gap by constructing a Coulomb gauge for any stationary admissible YangMills connection with small $L^{2}$-norm of curvature. This turns out to be highly non-trivial.

The cubes $[-1,2]^{n}$ and $[0,1]^{n}$ can of course be rescaled; however, one should caution that in the higher-dimensional case $n>4$, the energy $\int|F(A)|^{2}$ is not invariant under scaling. So if one were for instance to replace $[-1,2]^{n}$ by $[-r, 2 r]^{n}$ in (4), then the right-hand side should be replaced by $\varepsilon r^{n-4}$. It will also be clear from the proof that the underlying space $\mathbf{R}^{n}$ can be replaced by a smooth $n$-dimensional manifold.

Let $A$ be any stationary admissible Yang-Mills connection. By the monotonicity formula of Price [2], the integral $r^{4-n} \int_{B(x, r)}\left|F_{A}\right|^{2}$ is non-decreasing. So the density function

$$
\Theta(A, x)=\lim _{r \rightarrow 0} r^{4-n} \int_{B(x, r)}\left|F_{A}\right|^{2}
$$

\footnotetext{
${ }^{2}$ Here and in the sequel, $C$ denotes various absolute constants depending only on $n, G$, and $N$.

${ }^{3}$ This was pointed out to the second author by K. Uhlenbeck.
} 
exists for any $x \in \Omega$. The above theorem implies that $x$ is a singularity of $A$ modulo all gauge transformations if and only if $\Theta(A, x) \geq \epsilon$. In fact, one can give a better lower bound for the density at a genuine singular point $x$ :

$$
\Theta(A, x) \geq \min \left\{\inf _{B} c_{n, 4} \int_{S^{4}}\left|F_{B}\right|^{2}, \inf _{5 \leq k \leq n} \inf _{B^{\prime}} \frac{c_{n, k}}{k-4} \int_{S^{k-1}}\left|F_{B^{\prime}}\right|^{2}\right\},
$$

where $B$ and $B^{\prime}$ run over all non-flat Yang-Mills connections on $S^{4}$ and $S^{k-1}$, respectively, and furthermore,

$$
c_{n, k}=\int_{B^{n-k}(0,1)}\left(\sqrt{1-r^{2}}\right)^{k-4} d v
$$

where $B^{n-k}(0,1)$ denotes the unit ball in $\mathbf{R}^{n-k}$. Its proof can be outlined as follows. If $\Theta(A, x)$ is smaller than the given number, then it follows from results in [5] that modulo gauge transformations, by taking a subsequence if necessary, scaled connections $\lambda A(x+\lambda(y-x))$ converge to a Yang-Mills connection $A_{\infty}$ on $\mathbf{R}^{n} \backslash S$, which is simply the homogeneous extension of a Yang-Mills connection on $S^{n-1}$, where $S$ is a closed subset with $(n-4)$-dimensional Hausdorff measure zero and which is invariant under scalings. Then the claim follows from direct computations and induction on dimensions.

The proof of Theorem 1.1 is somewhat lengthy and proceeds in several stages, which we now describe.

The first step is to recall that stationary admissible Yang-Mills connections are smooth outside of a small set (a compact singular set $S$ of codimension at least four). Furthermore, thanks to Price's monotonicity formula 2, the curvature $F(A)$ is not only small in the $L^{2}$ norm (4), but also small in a certain Morrey space $M_{2}^{n / 2}\left([0,1]^{n}\right)$, defined below. This will be important because, in the higherdimensional case $n>4$, the $L^{2}$ norm is not scale invariant, but the Morrey norm is. It follows from this step that $\rho^{2} F(A)$ is uniformly bounded outside $S$; in fact, it is small near $S$, where $\rho$ denotes the distance from the set $S$.

Next we show that smooth connections with small $L^{2}$-norm of curvature can be placed in the Coulomb gauge $d_{*} A=0$, following the approach of Uhlenbeck 6 . This can be done because these smooth connections have small curvature in the Morrey norm. To do this we need to generalize Uhlenbeck's lemma on Coulomb gauges from Lebesgue spaces to Morrey spaces, which turns out to be relatively standard. This generalization has also been achieved independently by Rivière and Meyer [1]). It follows easily from this step that if a $W^{1,2}$-connection $A$ is weakly a limit of smooth connections with small $L^{2}$-norm of curvature, then $A$ has a Coulomb gauge.

In the next step, we excise the singular set $S$ by approximating the connection $A$ as a weak limit of smooth connections. The difficulty here is in ensuring that the smooth connections still have small curvature. If $S$ is a union of disjoint smooth submanifolds, one can construct such approximations by first eliminating the component of $A$ in the $\rho$-direction and using the curvature estimate in the first step 4 However, this problem turns out to be surprisingly non-trivial if we do not have a prior knowledge of the smooth structure of $S$. We will proceed by first performing an inductive gauge transform, averaging various radial gauges together, to transform the connection $A$ to one which obeys good bounds away from the singular

${ }^{4}$ We have obtained this as well as the last step long before we could do the next. 
set $S$ (roughly speaking, we need a connection which blows up like $\varepsilon / \operatorname{dist}(x, S)$ ). We then truncate this transformed connection by a cutoff function to obtain the approximating connections.

These last two steps are the main technical parts of this paper.

Finally, by taking limits, we can conclude from the above two steps that the original Yang-Mills connection $A$ can be placed in the Coulomb gauge. At this point one can use the Yang-Mills equation and some standard elliptic theory to obtain the desired regularity of $A$, even across the singular set $S$. This was already done in 5 in a different way.

\section{Notation}

In this section we lay out some notation, especially relating to the Lie group $G$, and the relationships between connections $A$, curvatures $F$, and gauge transforms $\sigma$. A useful heuristic5 to keep in mind is that the curvature acts like one derivative of the connection, which in turn acts like one derivative of the gauge transform (cf. (2), (1)).

In this paper we use $C$ to denote various constants which depend only on the ambient dimension $n$, the Lie group $G$, and the dimension $N$ of the unitary group $U(N)$ containing $G$. We use $A \sim B$ to denote the estimate $C^{-1} A \leq B \leq C A$.

We use $B(x, r):=\left\{y \in \mathbf{R}^{n}:|y-x|<r\right\}$ to denote the open ball of radius $r$ centered at $x$. If $E$ is a set in $\mathbf{R}^{n}$, we use $|E|$ to denote the Lebesgue measure of $E$; thus, for instance, $|B(x, r)|=C r^{n}$. Also note that if $0<r \leq 1$ and $x \in[0,1]^{n}$, then $\left|B(x, r) \cap[0,1]^{n}\right| \sim r^{n}$.

Recall that the Lie group $G$ is embedded in a unitary group $U(N)$, so that $\mathfrak{g}$ is embedded in the vector space $u(N)$. In particular, we have $\left|\sigma \cdot A \cdot \sigma^{-1}\right|=|A|$ for all $\sigma \in G$ and $A \in \mathfrak{g}$, where $|A|$ denotes the operator norm in $u(N)$. We use $1_{G}$ to denote the identity element in $G$.

If $A$ is a connection, we define $|A(x)|:=\left(\sum_{\alpha}\left|A_{\alpha}(x)\right|^{2}\right)^{1 / 2}$ and $|F(A(x))|:=$ $\left(\sum_{\alpha, \beta}\left|F_{\alpha \beta}(A)(x)\right|^{2}\right)^{1 / 2}$. From the identity

$$
F(\sigma(A))=\sigma F(A) \sigma^{-1}
$$

we observe that the magnitude $|F(A)|$ of the curvature is gauge invariant:

$$
|F(\sigma(A))|=|F(A)| \text {. }
$$

For future reference, we also record the composition law

$$
\sigma_{1}\left(\sigma_{2}(A)\right)=\left(\sigma_{1} \sigma_{2}\right)(A)
$$

\section{SOME PRELIMINARIES ON MORREy SPACES}

In this section we set up some basic notation, in particular the notation for Morrey spaces, and develop some of the basic functional theory for these spaces such as fractional integration, Sobolev embedding, etc.

\footnotetext{
${ }^{5}$ Related to this heuristic is the following dimensional analysis: if we give distances in $\mathbf{R}^{n}$ the scaling of length, then $\sigma$ has units of length $h^{0}, A$ has units of length ${ }^{-1}$, and $F$ has units of length ${ }^{-2}$, since each derivative in space effectively has the units of length ${ }^{-1}$. Meanwhile, integration on $n$-dimensional sets (e.g., balls $B(x, r)$ or the cube $\left.[0,1]^{n}\right)$ effectively has units of length ${ }^{n}$, and constants such as $C$ and $\varepsilon$ are dimensionless. The reader may then check that all of the estimates in this paper are dimensionally consistent.
} 
Suppose $A$ is a stationary Yang-Mills connection obeying the curvature smallness condition (4). By using Price's monotonicity formula [2] as in [5], we can improve (44) to the scale-invariant bounds

$$
\int_{B(x, r) \cap[0,1]^{n}}|F(A)|^{2} \leq C \varepsilon r^{n-4}
$$

for all balls $B(x, r)$. This is equivalent to a Morrey space estimate on $F(A)$, and motivates introducing the following (standard) notation.

We follow the notation of [4]:

Definition 3.1. If $\Omega$ is a domain and $1 \leq q \leq p$, we define the Morrey spaces $M_{q}^{p}(\Omega)$ to be those locally $L^{q}$ functions (possibly vector-valued) whose norm

$$
\|f\|_{M_{q}^{p}}:=\sup _{x_{0} \in \mathbf{R}^{n} ; 0<r \leq 1} r^{n\left(\frac{1}{p}-\frac{1}{q}\right)}\left(\int_{B\left(x_{0}, r\right) \cap \Omega}|f|^{q}\right)^{1 / q}
$$

is finite. We also define Morrey-Sobolev spaces $M_{q, k}^{p}$ for integers $k \geq 0$ by the formula

$$
\|f\|_{M_{q, k}^{p}(\Omega)}:=\sum_{j=0}^{k}\left\|\nabla^{j} f\right\|_{M_{q}^{p}(\Omega)} .
$$

In practice $k$ shall always be 0,1 , or 2 .

Thus Price's monotonicity formula gives $M_{2}^{n / 2}\left([0,1]^{n}\right)$ control on $F(A)$.

The norm $M_{q}^{p}$ has the scaling of $L^{p}$, but the functions are only $L^{q}$ integrable. From Hölder's inequality we see that all $L^{p}$ functions are in $M_{q}^{p}$, but not conversely. Note that the $M_{q}^{p}$ norm depends only on the magnitude of $f$. In particular, we see from (10) that

$$
\|F(\sigma(A))\|_{M_{q}^{p}}=\|F(A)\|_{M_{q}^{p}} .
$$

This gauge invariance of the Morrey norms for curvatures will be extremely handy in our analysis.

From Definition [3.1 and Hölder's inequality we see in particular that

$$
\frac{1}{|B(x, r)|} \int_{B(x, r) \cap \Omega}|f| \leq C r^{-n / p}\|f\|_{M_{q}^{p}(\Omega)} .
$$

In other words, if $f \in M_{q}^{p}(\Omega)$, then $f$ has magnitude $O\left(r^{-n / p}\right)$ on balls of radius $r$, in some $L^{q}$-averaged sense.

We now develop some basic estimates on Morrey spaces. All our functions here will be assumed to be smooth; it will not make a difference whether the functions are scalar, vector, or 2-form valued since we are allowing our constants $C$ to depend on $N$. In this section we shall also allow the constants $C$ to depend on the exponents $p, q$.

From Hölder's inequality we have

$$
\|f g\|_{M_{q}^{p}(\Omega)} \leq C\|f\|_{M_{q_{1}}^{p_{1}}(\Omega)}\|g\|_{M_{q_{2}}^{p_{2}}(\Omega)}
$$

for arbitrary $f, g, \Omega$, whenever $1 / p=1 / p_{1}+1 / p_{2}$ and $1 / q=1 / q_{1}+1 / q_{2}$. In particular, if $\Omega$ has finite measure, then $M_{q_{1}}^{p_{1}}(\Omega)$ embeds into $M_{q}^{p}(\Omega)$. Also we have

$$
\|f g\|_{M_{q}^{p}(\Omega)} \leq C\|f\|_{M_{q}^{p}(\Omega)}\|g\|_{L^{\infty}(\Omega)},
$$


and that the dual of $M_{q}^{p}$ is $M_{q^{\prime}}^{p^{\prime}}$ when $1<q \leq p<\infty$. Finally, we have the trivial observation

$$
\left\|\nabla^{j} f\right\|_{M_{q, k}^{p}(\Omega)} \leq C\|f\|_{M_{q, k+j}^{p}(\Omega)} .
$$

We shall use the above estimates so frequently in the sequel that we shall not explicitly mention them again.

We now develop analogues of standard harmonic analysis estimates for the Morrey space setting.

Proposition 3.2. Let $1<q \leq p<\infty$, and let $T$ be a pseudo-differential operator of order 0 . Then $T$ is bounded on $M_{q}^{p}\left(\boldsymbol{R}^{n}\right)$.

The same result holds if $T$ is replaced by the Hardy-Littlewood maximal operator

$$
\mathrm{M} u(x):=\sup _{r>0} \frac{1}{|B(x, r)|} \int_{B(x, r)}|u| .
$$

Proof. We give the proof for $T$ only, as the argument for M is completely analogous.

We need to show that

$$
r^{n\left(\frac{1}{p}-\frac{1}{q}\right)}\|T f\|_{L^{q}(B(x, r))} \leq C\|f\|_{M_{q}^{p}\left(\mathbf{R}^{n}\right)}
$$

for all balls $B(x, r)$. By scaling we may take $B(x, r)=B(0,1)$.

First suppose that $f$ is supported on $B(0,2)$. Then the claim follows from the standard result that $T$ is bounded on $L^{q}$ (see e.g. [3]). Thus we may assume that $f$ vanishes on $B(0,2)$. In this case we use the fact that the kernel $K(x, y)$ of $T$ must decay like $O\left(|x-y|^{n}\right)$ and a standard dyadic decomposition to obtain the pointwise estimate

$$
|T f(x)| \leq C \sum_{k=0}^{\infty} 2^{-n k} \int_{B\left(0,2^{k}\right)}|f| .
$$

The claim then follows from (13).

As an immediate corollary of this proposition we see that any smoothing operator of order $k$ will map $M_{q}^{p}$ to $M_{q, k}^{p}$ whenever $1<q \leq p<\infty$.

We now develop further corollaries of the above proposition.

Proposition 3.3 (Fractional integration). Whenever $n / 2 \leq p<n$ and $1 / q=$ $1 / p-1 / n$, we have

$$
\left\|u * \frac{1}{|x|^{n-1}}\right\|_{M_{4}^{q}\left(\boldsymbol{R}^{n}\right)} \leq C_{p, q}\|u\|_{M_{2}^{p}\left(\boldsymbol{R}^{n}\right)}
$$

Proof. We may assume that $\|u\|_{M_{2}^{p}\left(\mathbf{R}^{n}\right)}=1$.

Let $x_{0}$ be an arbitrary point in $\mathbf{R}^{n}$, and use dyadic decomposition and Hölder's inequality to estimate

$$
\begin{aligned}
\left|u * \frac{1}{|x|^{n-1}}\left(x_{0}\right)\right| \leq & \sum_{k \in \mathbf{Z}: 2^{k}<r} 2^{-(n-1) k}\|u\|_{L^{1}\left(B\left(x_{0}, 2^{k}\right)\right)} \\
& +\sum_{k \in \mathbf{Z}: 2^{k} \geq r} 2^{-(n-1) k} 2^{n k / 2}\|u\|_{L^{2}\left(B\left(x_{0}, 2^{k}\right)\right)}
\end{aligned}
$$

where $r>0$ will be chosen later.

We can bound the first term by $C r \mathrm{M} u\left(x_{0}\right)$. To control the second term, we use the bound $\|u\|_{L^{2}\left(B\left(x_{0}, 2^{k}\right)\right)} \leq C_{p} r^{n\left(\frac{1}{2}-\frac{1}{p}\right)}$ and the assumption $p<n$ to control this 
by $r^{1-n / p}$. Adding the two estimates together and optimizing in $r$ we thus have (after some algebra) the pointwise estimate

$$
\left|u * \frac{1}{|x|^{n-1}}\left(x_{0}\right)\right| \leq C_{p} \mathrm{M} u\left(x_{0}\right)^{q / p} .
$$

Thus

$$
\left\|u * \frac{1}{|x|^{n-1}}\right\|_{M_{4}^{q}} \leq C_{p}\|\mathrm{M} u\|_{M_{4 p / q}^{p}}^{q / p} .
$$

Since $p \leq n / 2$ and $1 / q=1 / p-1 / n$, we have $4 p / q \leq 2$. The claim then follows from Proposition 3.2

We now specialize our domain $\Omega$ to the unit cube $[0,1]^{n}$.

Corollary 3.4 (Morrey-Sobolev embeddings). We have the estimate

$$
\|u\|_{M_{4}^{q}\left([0,1]^{n}\right)} \leq C_{p, q}\|u\|_{M_{2,1}^{p}\left([0,1]^{n}\right)}
$$

whenever $n / 2 \leq p<n$ and $1 / q=1 / p-1 / n$. If $p$ is strictly greater than $n / 2$, we also have

$$
\|u\|_{L^{\infty}\left([0,1]^{n}\right)} \leq C\|u\|_{M_{2,2}^{p}\left([0,1]^{n}\right)} ;
$$

in fact, we can replace $L^{\infty}$ by the Hölder space $C^{0, \alpha}$ for some $0<\alpha=\alpha(p)<1$.

Proof. We first prove (14). From the fundamental theorem of calculus and polar coordinates around $x_{0}$ we have the pointwise estimate

$$
\left|u\left(x_{0}\right)\right| \leq C\left(|\nabla u| *|x|^{1-n}\right)\left(x_{0}\right)+\int_{[0,1]^{n}}|u| .
$$

The former term is acceptable by Proposition 3.3. The latter term is acceptable by (13).

The claim (15) (and the Hölder refinement) then follows from (14) and Morrey's lemma; see, e.g., 4].

From the above proposition and Hölder's inequality we obtain the basic estimate

$$
\|u v\|_{M_{2}^{n / 2}\left([0,1]^{n}\right)} \leq C\|u\|_{M_{2,1}^{n / 2}\left([0,1]^{n}\right)}\|v\|_{M_{2,1}^{n / 2}\left([0,1]^{n}\right)^{\prime}}
$$

This estimate is what allows us to control the non-linear portion $A \wedge A$ of the curvature by the linear portion $d A$, assuming that one is in the Coulomb gauge and that the curvature is small in $M_{2}^{n / 2}$.

For technical reasons having to do with continuity arguments we shall need to also work in slightly smoother spaces than $M_{2, k}^{n / 2}$, and in particular we shall need to investigate the continuity of the Coulomb gauge construction in the space $M_{2,1}^{p}$ for some $n / 2<p<n$.

For these spaces one can use Corollary [3.4. Hölder's inequality, and the Leibnitz rule for derivatives to obtain the product estimates

$$
\begin{aligned}
\|u v\|_{M_{2, i+j-2}^{p}\left([0,1]^{n}\right)} \leq C_{p}\|u\|_{M_{2, i}^{p}\left([0,1]^{n}\right)}\|v\|_{M_{2, j}^{p}\left([0,1]^{n}\right)} & \\
& \text { whenever } i, j, i+j-2 \in\{0,1,2\} .
\end{aligned}
$$

Also, we shall need the variant

$$
\|u v\|_{M_{2}^{p}\left([0,1]^{n}\right)} \leq C_{p}\|u\|_{M_{2,1}^{p}\left([0,1]^{n}\right)}\|v\|_{M_{2,1}^{n, 2}\left([0,1]^{n}\right)},
$$

which is proven by the same methods. 
We now give some standard elliptic regularity estimates for Morrey spaces. In proving these estimates it is convenient to define the approximate fundamental solution $K$ to the Laplacian by

$$
K:=c \phi /|x|^{2-n}
$$

where $\phi$ is a smooth radial bump function which equals 1 on $[-2,2]^{n}$ and $c:=$ $-4 \pi^{n / 2} / \Gamma\left(\frac{n-2}{2}\right)$ is the constant such that $\Delta \frac{c}{|x|^{2-n}}$ is the Dirac delta. Observe that $\Delta K=\delta+\psi$ for some bump function $\psi$.

Proposition 3.5 (Elliptic regularity). Let $1<q<\infty$. If $u^{\alpha}, \varphi^{\alpha \beta}$ satisfy the Hodge system

$$
\begin{aligned}
\partial^{\beta} u^{\alpha}-\partial^{\alpha} u^{\beta} & =\varphi^{\alpha \beta}, \\
\partial_{\alpha} u^{\alpha} & =0
\end{aligned}
$$

on $[0,1]^{n}$ with the boundary condition

$$
n_{\alpha} u^{\alpha}=0
$$

on $\partial[0,1]^{n}$, then

$$
\|u\|_{M_{2,1}^{q}\left([0,1]^{n}\right)} \leq C_{q}\|\varphi\|_{M_{2}^{q}\left([0,1]^{n}\right)} .
$$

Proof. Extend the one-form $u^{\beta}$ and the two-form $\varphi^{\alpha \beta}$ to $\mathbf{R}^{n}$ so that they are symmetric with respect to reflections across the faces of $[0,1]^{n}$. Observe from (19), (20), (21) that (19), (20) in fact hold on all of $\mathbf{R}^{n}$ in the sense of distributions.

Contracting (19) against $\partial_{\beta}$ and using (20), we obtain

$$
\Delta u^{\alpha}=\partial_{\beta} \varphi^{\alpha \beta} .
$$

Motivated by this, we define

$$
\tilde{u}^{\alpha}:=\partial_{\beta} \varphi^{\alpha \beta} * K .
$$

We thus see that $\Delta\left(u^{\alpha}-\tilde{u}^{\alpha}\right)$ is smooth on $[0,1]^{n}$, and that $n_{\alpha}\left(u^{\alpha}-\tilde{u}^{\alpha}\right)=0$ on $\partial[0,1]^{n}$. From this and standard elliptic regularity one sees that the contribution of $u-\tilde{u}$ is acceptable. To deal with the contribution of $\tilde{u}$ we just observe that convolution with $\partial_{\beta} K$ is a standard smoothing operator of order 1 , and use Proposition 3.2.

Proposition 3.6 (Neumann problem regularity). Let $1<q<\infty$. Let $u, f, g_{\beta} \in$ $C^{\infty}\left([0,1]^{n}\right)$ be such that

$$
\Delta u=f
$$

on $(0,1)^{n}$ and

$$
n^{\beta} \partial_{\beta} u=n^{\beta} g_{\beta}
$$

on $\partial[0,1]^{n}$, and we have the normalization

$$
\int_{[0,1]^{n}} u=0 .
$$

Then we have

$$
\|u\|_{M_{2,2}^{q}\left([0,1]^{n}\right)} \leq C\|f\|_{M_{2}^{q}\left([0,1]^{n}\right)}+C\|g\|_{M_{2,1}^{q}\left([0,1]^{n}\right)} .
$$


Proof. We first prove the claim when $f=0$. From the Sobolev trace lemma we observe that $g_{\beta}$ is in $L^{2}$ on hyperplanes.

For each $\beta$, we extend $g_{\beta}$ to the domain $\left\{x: x_{\beta} \in[0,1]\right\}$ by requiring $g_{\beta}$ to be symmetric with respect to reflections along the faces of $[0,1]^{n}$ perpendicular to $e_{\beta}$. Define $\tilde{u}$ on $[0,1]^{n}$ by

$$
\tilde{u}(x):=\sum_{\beta=1}^{n} \int_{y_{\beta}=1} K(x-y) g_{\beta}(y) d y-\int_{y_{\beta}=0} K(x-y) g_{\beta}(y) d y .
$$

Since $\Delta K=\delta+\psi$ we have

$$
\Delta \tilde{u}(x)=\sum_{\beta=1}^{n} \int_{y_{\beta}=1} \psi(x-y) g_{\beta}(y) d y-\int_{y_{\beta}=0} \psi(x-y) g_{\beta}(y) d y
$$

on $(0,1)^{n}$. In particular, $\Delta \tilde{u}$ is smooth, with a norm controlled by the $M_{2,1}^{q}\left([0,1]^{n}\right)$ norm of $g$. From the Plemelj formulae and symmetry we also see that $n^{\beta} \partial_{\beta} \tilde{u}=n^{\beta} g_{\beta}$ on $\partial[0,1]^{n}$. Thus, it remains only to show that $\tilde{u}$ is in $M_{2,2}^{q}\left([0,1]^{n}\right)$, since the difference $u-\tilde{u}$ can be controlled easily by classical Neumann theory.

By symmetry it suffices to show that

$$
\left\|\int_{y_{n}=0} K(x-y) g(y) d y\right\|_{M_{2,2}^{q}\left([0,1]^{n}\right)} \leq C\|g\|_{M_{2,1}^{q}\left(\mathbf{R}^{n}\right)}
$$

for any function $g$ on $\mathbf{R}^{n}$. By applying a cutoff we may assume that $g(y)$ is supported on the region $\left|y_{n}\right| \leq 1$. We may then invoke the fundamental theorem of calculus and polar coordinates to write

$$
g=\nabla g * L
$$

for some (covector-valued) kernel $L$ supported on the cone $\left\{x:|x| \leq x_{n} \leq C\right\}$ which behaves like $|x|^{1-n}$. It thus suffices to show that

$$
\left\|\nabla_{x}^{k} \iint_{y_{n}=0} K(x-y) L(y-z) G(z) d y d z\right\|_{M_{2,2}^{q}\left([0,1]^{n}\right)} \leq C\|G\|_{M_{2}^{q}\left(\mathbf{R}^{n}\right)}
$$

for all $G$ and $k=0,1,2$.

The expression inside the norm vanishes for $\left|y_{n}\right| \geq C$. From this and the fundamental theorem of calculus we see that we need only prove the above estimate for $k=2$.

A computation shows that

$$
\left|\nabla_{x}^{2} \int_{y_{n}=0} K(x-y) L(y-z) d y\right| \leq x_{n}^{-n}\left(1+|z-x| / x_{n}\right)^{-n-1}
$$

for all $x, z$, which implies the pointwise estimate

$$
\left|\nabla_{x}^{k} \iint_{y_{n}=0} K(x-y) L(y-z) G(z) d y d z\right| \leq C \mathrm{M} G(x) .
$$

The claim then follows from Proposition 3.2 .

We now consider the general case when $f$ is not necessarily 0 . Define

$$
v:=u-\left(f \chi_{[0,1]^{n}}\right) * K .
$$

Then

on $[0,1]^{n}$ and

$$
\partial_{\alpha} \partial^{\alpha} v=0
$$

$$
n^{\beta} \partial_{\beta} v=n^{\beta}\left(g_{\beta}-f \chi_{[0,1]^{n}} * \partial_{\beta} K\right) .
$$


The operation of convolution with $K$ is smoothing of order 2 ; thus,

$$
\left\|\left(f \chi_{[0,1]^{n}}\right) * K\right\|_{M_{2,2}^{q}} \leq C\|f\|_{M_{2}^{q}}
$$

and (26) then follows from the $f=0$ estimate applied to $v$.

Lemma 3.7 (Interior regularity). Let $B(x, r)$ be a ball, and let $0<\theta \ll 1$. Then we have

$$
\|u\|_{M_{2,1}^{n / 2}(B(x, \theta r))} \leq C\|\Delta u\|_{M_{4 / 3}^{3 n / 2}(B(x, r))}+C \theta^{2}\|u\|_{M_{2,1}^{n / 2}(B(x, r))}
$$

whenever $u$ is such that the right-hand side makes sense.

Proof. By the usual limiting argument (using the ellipticity of $\Delta$ ) we may assume that $u$ is smooth. We may rescale $B(x, r)$ to be $B(0,1)$.

Let $\eta$ be a bump function adapted to $B(0,1)$ which equals 1 on $B(0,1 / 2)$. We split

$$
u=K *(\eta \Delta u)+(u-K *(\eta \Delta u)) .
$$

Consider the latter term. This is harmonic on $B(0,1 / 2)$. So by interior regularity we have

$$
\left\|\nabla^{j}(u-K *(\eta \Delta u))\right\|_{L^{\infty}(B(0, \theta))} \leq C\|u-K *(\eta \Delta u)\|_{L^{1}(B(0,1 / 4))}
$$

for $j=0,1$. The right-hand side can be easily bounded by

$$
C\|\Delta u\|_{M_{4 / 3}^{3 n / 2}(B(0,1))}+C\|u\|_{M_{2,1}^{n / 2}(B(0,1))} .
$$

Since for any $F$ we have

$$
\|F\|_{M_{2}^{n / 2}(B(0, \theta))} \leq C \theta^{2}\|F\|_{L^{\infty}(B(0, \theta))},
$$

we thus see that the contribution of this term is acceptable.

To control the first term it suffices to show the global estimate

$$
\left\|\nabla^{j} K * f\right\|_{M_{2}^{n / 2}\left(\mathbf{R}^{n}\right)} \leq C\|f\|_{M_{4 / 3}^{3 n / 2}\left(\mathbf{R}^{n}\right)}
$$

for test functions $f$ and $j=0,1$. But this follows from the dual of Proposition 3.3 (with $p=n / 2$ and $q=n$ ), since $\nabla^{j} K$ is pointwise bounded by $C /|x|^{n-1}$.

\section{Overview of Proof of Theorem 1.1}

We now give the proof of Theorem 1.1, modulo some propositions which we will prove in later sections.

Fix $A$ to be an admissible Yang-Mills connection obeying the assumptions in Theorem 1.1. From the analysis in [5] we have the following properties:

Proposition 4.1. Let $A$ be a stationary admissible Yang-Mills connection and $S$ be its singular set. Then the curvature $F(A)$ obeys the Morrey norm estimate

$$
\|F(A)\|_{M_{2}^{n / 2}\left([0,1]^{n}\right)} \leq C \varepsilon
$$

and the pointwise bounds

$$
|F(A)(x)| \leq \frac{C \varepsilon}{\rho(x)^{2}} \text { for all } x \in[0,1]^{n} \backslash S,
$$

where $\rho(x)$ is the distance function

$$
\rho(x):=\operatorname{dist}(x, S) .
$$


Inequality (27) follows from the monotonicity for stationary Yang-Mills connections. Inequality (28) is obtained by applying curvature estimates to the smooth connection $A$ outside $S$ and using (27). The singular set is extremely small, having codimension at least 4 . This high codimension shall be crucial in our arguments, as it allows various path and surface integrals to be generically well-defined. Note that we have no control over the topology or regularity of $S$ (other than that $S$ is compact); however, we will use averaging arguments to get around this difficulty.

We remark that the next few steps of the argument (Lemma 4.2. Proposition 4.3. Proposition 4.4, Theorem 4.6) do not use the Yang-Mills equation (3) directly; instead, they proceed from the conclusions in Proposition 4.1, which of course hold for a more general class of connections than the stationary admissible Yang-Mills connections. The Yang-Mills equation only reappears much later in the argument, in Lemma 4.7

The function $F(A)$ is defined a.e. on $[0,1]^{n}$; we extend it by zero outside of this unit cube. From (27) and (13) we observe in particular that

$$
\int_{B(x, r)}|F(A)| \leq C \varepsilon r^{n-2}
$$

for all balls $B(x, r)$.

To visualize a function $F(A)$ which obeys (27), (28), one model to keep in mind is when $S$ is a smooth $(n-4)$-dimensional surface, and $|F(A)(x)| \sim \varepsilon / \rho(x)^{2}$. (This function is not quite in $L^{2}$, and so (27) is not quite obeyed, but this can be rectified by adding a logarithmic decay factor.) Using the heuristic that the connection $A$ is like an antiderivative of the curvature $F(A)$, one then expects to be able to place $A$ in a gauge $\sigma(A)$ so that $|\sigma(A)(x)| \leq C \varepsilon / \rho(x)$. Another formulation of this heuristic is that one expects to be able to find a gauge $\sigma(A)$ obeying the pointwise bounds

$$
|\sigma(A)(x)| \leq C \int_{[0,1]^{n}} \frac{|F(A)(y)|}{|x-y|^{n-1}} d y,
$$

since the right-hand side is essentially the fractional integral $|\nabla|^{-1}|F(A)|$ of $|F(A)|$. Such a gauge is easy to obtain in the case when $G$ is abelian, since one can use Hodge theory to find a gauge $\sigma(A)$ which is essentially equal to $\Delta^{-1} d_{*} F \approx \nabla^{-1} F$. An essentially equivalent approach in the abelian case is to take various radial gauges (where $\sigma(A)(x) \cdot\left(x-x_{0}\right)=0$ for all $x \in[0,1]^{n}$ ) and average over all choices of origin $x_{0} \in[0,1]^{n}$ to obtain a connection obeying the bounds (30). Note that this averaging will eliminate the difficulty that the rays in the radial gauge occasionally pass through the singular set $S$, since this set has such high codimension.

We do not know how to achieve bounds of the form (30) in the non-abelian case, however we can develop a reasonably good rigorous substitute for this heuristic, which we now discuss. The idea is to inductively construct a successive sequence of partial gauges which obey reasonable connection bounds and which advance closer and closer to the singular set $S$ as the induction progresses. 
We shall need a fixed exponent $0<\kappa<1$; for the sake of concreteness we set $\kappa:=1 / 2$. We define the quantity $Q(x)$ on $\mathbf{R}^{n}$ by

$$
Q(x):=\sup _{0<r \leq \operatorname{diam}\left([0,1]^{n}\right)} r^{-n / 2+1+\kappa}\left(\int_{B(x, r)}|F(A)(y)|^{2} d y\right)^{1 / 2} ;
$$

this expression is like the Hardy-Littlewood maximal function $\mathrm{M} F(A)$ but with an additional decay factor of $r^{1+\kappa}$. In the model case where $S$ is a smooth $(n-4)$ dimensional surface and $F$ is comparable to $\varepsilon / \rho(x)^{2}$, the quantity $Q(x)$ is comparable to $\varepsilon / \rho(x)^{1-\kappa}$; the reader may find this model case helpful to keep in mind in what follows.

Let $D \gg 1$ be a large number depending only on $n, G, N$ to be chosen later; we will assume that $\varepsilon$ is sufficiently small depending on $D$. This quantity $D$ shall be our dyadic base, i.e., we will use powers $D^{m}$ of $D$ to define dyadic scales, as opposed to the more usual powers of two.

We define the domains $\Omega_{m}$ for $m=1,2, \ldots$ by

$$
\Omega_{m}:=\left\{x \in[0,1]^{n} \backslash S: Q(x)<\varepsilon D^{(1-\kappa) m}\right\} .
$$

Thus in the model case, $\Omega_{m}$ is essentially the region where $\rho(x) \geq D^{-m}$.

In general, the $\Omega_{m}$ are a non-decreasing collection of open sets. As $m$ gets larger, the set $\Omega_{m}$ fills out an increasingly large portion of the unit cube, as the following lemma indicates:

Lemma $4.2\left(\Omega_{m}\right.$ is dense at scale $\left.R_{m}\right)$. For any $m \geq 1$, define the radius

$$
R_{m}:=C D^{-m}
$$

where $C$ is a sufficiently large constant. Then we have

$$
\left\{x \in[0,1]^{n} \backslash S: \rho(x) \geq R_{m}\right\} \subseteq \Omega_{m} .
$$

Furthermore, we have

$$
\left|B(x, r) \cap \Omega_{m}\right| \geq C^{-1} r^{n} \text { whenever } r \geq R_{m}, x \in[0,1]^{n} .
$$

Proof. We first prove (34). Let $x \in[0,1]^{n}$ be such that $\rho(x) \geq R_{m}$. We have to show that

$$
r^{-n / 2+1+\kappa}\left(\int_{B(x, r)}|F(A)(y)|^{2} d y\right)^{1 / 2} \leq \varepsilon D^{(1-\kappa) m}
$$

for all $0<r \leq \operatorname{diam}([0,1])$.

First suppose that $r<\rho(x) / 2$. Then by (28)) we can bound the left-hand side by

$$
r^{-n / 2+1+\kappa} r^{n / 2} \varepsilon / \rho^{2}(x) \leq C \varepsilon \rho(x)^{-1+\kappa} \leq C \varepsilon R_{m}^{-1+\kappa},
$$

which will be acceptable if the constant in (33) is large enough.

Now suppose that $r>\rho(x) / 2$. Then by 27 we can bound the left-hand side by

$$
r^{-n / 2+1+\kappa} \varepsilon r^{n / 2-2} \leq C \varepsilon \rho(x)^{-1+\kappa} \leq C \varepsilon R_{m}^{-1+\kappa},
$$

which is again acceptable.

\footnotetext{
${ }^{6}$ We apologize for the artificiality of the quantity $Q(x)$ and the companion quantity $T_{m}(x)$ defined in (37); these choices were obtained after much trial and error. One needs $Q$ small enough that one has the density bounds in Lemma 4.2 and the clustering bounds in Lemma 7.3 but $Q$ large enough that the errors arising from the truncation argument in Proposition 4.4 are manageable. For purposes of dimensional analysis, $Q$ has units of length ${ }^{\kappa-1}, T_{m}$ has units of length ${ }^{-1}$, while $\rho(x)$ and radii such as $r$ or $R_{m}=C D^{-m}$ have the units of length. This dimensional analysis can serve to explain many of the strange powers of $r$ or $R_{m}$ which appear in many of the estimates in the next few sections.
} 
Now we prove (35). Fix $B(x, r)$. For each $x^{\prime}$ in the compact set $\overline{B(x, r)} \backslash \Omega_{m}$ we see, from (32), (31), that there exists a radius $0<r\left(x^{\prime}\right) \leq C$ such that

$$
r\left(x^{\prime}\right)^{-n / 2+1+\kappa}\left(\int_{B\left(x^{\prime}, r\left(x^{\prime}\right)\right)}|F(A)(y)|^{2} d y\right)^{1 / 2} \geq \varepsilon D^{(1-\kappa) m} .
$$

From (27) we must have $r\left(x^{\prime}\right) \leq C D^{-m}$. In particular, we have $B\left(x^{\prime}, r\left(x^{\prime}\right)\right) \subseteq$ $B(x, 2 r)$ if the constant in (33) is large enough.

The balls $B\left(x^{\prime}, r\left(x^{\prime}\right)\right)$ clearly cover the compact set $\overline{B(x, r)} \cap\left([0,1]^{n} \backslash \Omega_{m}\right)$; so, in particular, there is a finite sub-cover of this set by these balls. By the Vitali covering lemma there thus exists a finite sub-collection $B\left(x_{j}, r\left(x_{j}\right)\right)$ of balls which are disjoint and such that $B\left(x_{j}, 5 r\left(x_{j}\right)\right)$ covers $\overline{B(x, r)} \cap\left([0,1]^{n} \backslash \Omega_{m}\right)$. In particular, we have

$$
\left|B(x, r) \cap[0,1]^{n} \backslash \Omega_{m}\right| \leq C \sum_{j} r\left(x_{j}\right)^{n} .
$$

On the other hand, by construction we have

$$
\int_{B\left(x_{j}, r\left(x_{j}\right)\right)}|F(A)(y)|^{2} d y \geq \varepsilon^{2} D^{2(1-\kappa) m} r\left(x^{\prime}\right)^{n-2-2 \kappa} \geq C^{-1} \varepsilon^{2} D^{4 m} r\left(x^{\prime}\right)^{n}
$$

since $r\left(x^{\prime}\right) \leq C D^{-m}$. Thus we have

$$
\left|B(x, r) \cap[0,1]^{n} \backslash \Omega_{m}\right| \leq \sum_{j} \frac{C}{\varepsilon^{2} D^{4 m}} \int_{B\left(x_{j}, r\left(x_{j}\right)\right)}|F(A)(y)|^{2} d y
$$

since the balls $B\left(x_{j}, r\left(x_{j}\right)\right)$ are disjoint and lie in $B(x, 2 r)$ we thus have

$$
\left|B(x, r) \cap[0,1]^{n} \backslash \Omega_{m}\right| \leq \frac{C}{\varepsilon^{2} D^{4 m}} \int_{B(x, 2 r)}|F(A)(y)|^{2} d y ;
$$

from (27) we thus have

$$
\begin{aligned}
\left|B(x, r) \cap[0,1]^{n} \backslash \Omega_{m}\right| \leq C D^{4 m} r^{n-4} & \leq C\left|B(x, r) \cap[0,1]^{n}\right|\left(r D^{m}\right)^{-4} \\
& \leq C\left|B(x, r) \cap[0,1]^{n}\right|\left(r / R_{m}\right)^{-4} .
\end{aligned}
$$

If the constant in (33) is sufficiently large, the claim (35) follows.

We are now ready to state the precise analogue of the heuristic (30).

Proposition 4.3. Let $A, S$ be as in Proposition 4.1. Then, if $D$ is sufficiently large and $\varepsilon$ is sufficiently small depending on $D$, for every $m \geq 1$ there exists a gauge transform $\sigma_{m}$ on $\Omega_{m}$ which obeys the bounds

$$
\left|\sigma_{m}(A)(x)\right| \leq C(D) T_{m}(x)
$$

for all $x \in \Omega_{m}$, where $T_{m}(x)$ is the quantity

$$
T_{m}(x):=\int_{[0,1]^{n}}\left(1+\frac{|y-x|}{R_{m}}\right)^{\kappa / 2} \frac{|F(A)(y)|}{|x-y|^{n-1}} d y .
$$

Using (29) one can crudely bound $T_{m}(x)$ by $O\left(\varepsilon / R_{m}\right)$, but we will need the more precise structure of (37) in our truncation analysis later in this section. However, this crude bound already shows that $\sigma_{m}(A)$ is bounded on $\Omega_{m}$, and hence (by (2) and the smoothness of $A$ ) that $\sigma_{m}$ is locally Lipschitz on $\Omega_{m}$, although our Lipschitz bound of course depends on $m$.

We do not assert here that $\sigma_{m}$ is smooth; however, when we use this proposition later in this section we will be able to regularize $\sigma_{m}$ with little difficulty. 
The construction of the gauges $\sigma_{m}$ will be inductive, with the gauge $\sigma_{m+1}$ obtained from $\sigma_{m}$ by integrating the connection $A$ along curves, using the curvature bound (27) to compare integrals along two different curves, and averaging over a family of curves; we give the construction and prove the proposition in Sections 5-7. It may be possible to obtain a result like this more directly, perhaps by using the finer structure of the Yang-Mills equation, but we were unable to do so.

Using Proposition 4.3 and a truncation argument, we will prove the following approximation theorem.

Proposition 4.4 (Approximation by smooth connections). For any $m>0$, there exists a smooth connection $A_{m}$ on $[0,1]^{n}$ which is gauge equivalent to $A$ on the open set

$$
\left\{x \in[0,1]^{n}: \rho(x) \geq 20 R_{m}\right\}
$$

and which obeys the smallness condition

$$
\left\|F\left(A_{m}\right)\right\|_{M_{2}^{n / 2}\left([0,1]^{n}\right)} \leq C(D) \varepsilon .
$$

We shall prove this proposition in Section 8 .

The next step is to place the smooth connections $A_{m}$ in a Coulomb gauge.

Definition 4.5. A connection $A$ is said to be a Coulomb gauge on $\Omega$ if it satisfies the condition $d_{*} A=0$ on the interior of $\Omega$, and $A \cdot n=0$ on the boundary $\partial \Omega$.

From elliptic theory we expect Coulomb gauges to be quite regular; specifically, we expect $A$ to have one more derivative of regularity than $F(A)$. The question then arises: given an arbitrary connection $A$, under what conditions can we find a gauge equivalent Coulomb gauge $A_{\text {coulomb }}$ which has one more derivative of regularity than $F(A)$ ?

In [6] this problem was considered assuming that the curvature was in $L^{n / 2}$. In our applications we need to replace this space by the slightly larger space $M_{2}^{n / 2}$. Using the heuristic that a connection requires one more derivative than the curvature, and a gauge transform requires two more derivatives, we thus hope to place connections and gauge transforms in $M_{2,1}^{n / 2}$ and $M_{2,2}^{n / 2}$ respectively.

For any $\varepsilon>0$, let $\mathbf{U}_{\varepsilon}(\Omega)$ denote the set of all smooth connections on $\Omega$ which satisfy the bound

$$
\|F(A)\|_{M_{2}^{n / 2}(\Omega)} \leq \varepsilon .
$$

From (12) we observe that this space is invariant under gauge transformations.

In Section 9 we prove the following generalization of Uhlenbeck's lemma [6]:

Theorem 4.6 (Small curvature allows a Coulomb gauge). If $0<\varepsilon \ll 1$ is sufficiently small, then every connection $A$ in $\boldsymbol{U}_{\varepsilon}\left([0,1]^{n}\right)$ is gauge equivalent (via a smooth gauge transformation $\sigma$ ) to a smooth Coulomb gauge $A_{\text {coulomb }}$ which obeys the bound

$$
\left\|A_{\text {coulomb }}\right\|_{M_{2,1}^{n / 2}\left([0,1]^{n}\right)} \leq C\|F(A)\|_{M_{2}^{n / 2}\left([0,1]^{n}\right)} .
$$

This theorem has also been independently obtained by Rivière and Meyer [1]. Note that Theorem 4.6 does not require $A$ to be Yang-Mills, but it does require $A$ to be smooth. 
We apply Theorem 4.6 to the smooth gauges $A_{m}$ constructed in Proposition 4.4 We may thus find (if $\varepsilon$ is sufficiently small depending on $C_{0}, D$ ) a Coulomb gauge $A_{m, \text { coulomb }}:=\sigma_{m}^{\text {coulomb }}\left(A_{m}\right)$ such that

$$
\left\|A_{m, \text { coulomb }}\right\|_{M_{2,1}^{n / 2}\left([0,1]^{n}\right)} \leq C \varepsilon .
$$

By weak compactness, we may thus find a sequence $m_{j} \rightarrow 0$ such that $A_{m_{j}, \text { coulomb }}$ converges weakly in $M_{2,1}^{n / 2}\left([0,1]^{n}\right)$ to a function $A_{\text {coulomb }}$ such that

$$
\left\|A_{\text {coulomb }}\right\|_{M_{2,1}^{n / 2}\left([0,1]^{n}\right)} \leq C \varepsilon .
$$

By taking weak limits of the Coulomb gauges $A_{m_{j}, \text { coulomb }}$ we thus see that $A_{\text {coulomb }}$ is also a Coulomb gauge

Let $K$ be an arbitrary compact subset of $[0,1]^{n} \backslash S$. Then for $j$ sufficiently large, we have that $R_{m_{j}} \ll \operatorname{dist}(K, S)$, so that $A_{m_{j}, \text { coulomb }}$ is gauge equivalent to $A$ on $K$. Thus we may find a gauge $\bar{\sigma}_{j}$ on $K$ such that

$$
d \bar{\sigma}_{j}=\bar{\sigma}_{j} A-A_{m_{j}, \text { coulomb }} \bar{\sigma}_{j} .
$$

Since $A, A_{m_{j}, \text { coulomb }}$ are in $L^{2}$ uniformly in $j$, we thus see that the $\nabla \bar{\sigma}_{j}$ are in $L^{2}$ uniformly in $j$. By passing to a subsequence if necessary and using Rellich embedding we see that $\bar{\sigma}_{j}$ converges strongly in $L^{q}$ for some $q>2$ to some limiting function $\sigma \in L^{q}$. In particular, we see that $\sigma$ takes values in $G$. By taking limits of (43) we thus see that

$$
d \sigma=\sigma A-A_{\text {coulomb }} \sigma
$$

and in particular that $\sigma \in W^{r, 1}$ for some $r>1$. Thus $A$ and $A_{\text {coulomb }}$ are gauge equivalent on $K$, and hence (since $K$ was arbitrary) on $[0,1]^{n} \backslash S$.

To summarize, we have found a gauge $A_{\text {coulomb }}=\sigma(A)$ on $[0,1]^{n} \backslash S$ which is a Coulomb gauge and obeys the smallness assumption (42). To complete the proof of Theorem 1.1, it will suffice to show that $A_{\text {coulomb }}$ extends to be smooth on all of $[0,1]^{n}$.

We shall use standard elliptic regularity techniques. The first step is to obtain some bounds on $\Delta A_{\text {coulomb }}$, which presently is only defined in the sense of distributions.

Lemma 4.7. The distribution $\Delta A_{\text {coulomb }}$ is a locally integrable function; in fact, it lies in the space $M_{4 / 3}^{3 n / 2}\left([0,1]^{n}\right)$ and obeys the pointwise estimate

$$
\left|\Delta A_{\text {coulomb }}\right| \leq C\left|A_{\text {coulomb }}\right|\left|\nabla A_{\text {coulomb }}\right|+C\left|A_{\text {coulomb }}\right|^{3}
$$

almost everywhere in $[0,1]^{n}$.

\footnotetext{
${ }^{7}$ Observe from 42 that the $A_{m_{j}}$,coulomb are uniformly in the Sobolev space $W^{1,2}$, and so by Rellich embedding and the Sobolev trace lemma they converge strongly in $L^{2}$ on the boundary of $[0,1]^{n}$; thus we may legitimately take limits of the boundary condition $A_{m_{j}}$,coulomb $\cdot n=0$.
} 
Proof. First we work in $[0,1]^{n} \backslash S$. On this set, $A_{\text {coulomb }}$ is a Yang-Mills connection in the Coulomb gauge. So by (3) and the Coulomb gauge condition we have

$$
\begin{aligned}
\Delta A_{\text {coulomb }} & =d_{*} d A_{\text {coulomb }}+d d_{*} A_{\text {coulomb }} \\
& =d_{*} d A_{\text {coulomb }} \\
& =d_{*}\left(F\left(A_{\text {coulomb }}\right)\right)-d_{*}\left(A_{\text {coulomb }} \wedge A_{\text {coulomb }}\right) \\
& =*\left[A_{\text {coulomb }}, * F\left(A_{\text {coulomb }}\right)\right]-d_{*}\left(A_{\text {coulomb }} \wedge A_{\text {coulomb }}\right) \\
& =O\left(\left|F\left(A_{\text {coulomb }}\right)\right|\left|A_{\text {coulomb }}\right|+\left|\nabla A_{\text {coulomb }}\right|\left|A_{\text {coulomb }}\right|\right) \\
& =O\left(\left|\nabla A_{\text {coulomb }}\right|\left|A_{\text {coulomb }}\right|+\left|A_{\text {coulomb }}\right|^{3}\right)
\end{aligned}
$$

on $[0,1]^{n} \backslash S$. Note that $F\left(A_{\text {coulomb }}\right)$ is in $M_{2}^{n / 2}$ and $A_{\text {coulomb }}$ is in $M_{4}^{n}$ (by (42), (14)). So the above computations are justified in the sense of distributions. In particular, we see that (44) holds a.e. in $[0,1]^{n} \backslash S$, which by (14), (42), and Hölder implies that $\Delta A_{\text {coulomb }}$, when restricted to $[0,1]^{n} \backslash S$, is locally integrable and lies in the Morrey space $M_{4 / 3}^{3 n / 2}\left([0,1]^{n} \backslash S\right)$.

We are almost done; however, we still have to exclude the technical possibility that the distribution $\Delta A_{\text {coulomb }}$ has a singular component on the set $S$. Fortunately, the high codimension of $S$ will prevent this from happening, as $S$ is too small to support singularities with the required regularity.

To avoid confusion, let us use $\left.\Delta A_{\text {coulomb }}\right|_{[0,1]^{n} \backslash S}$ to denote the (classical) Laplacian of $A_{\text {coulomb }}$ outside of $S$; we have already shown that $\left.\Delta A_{\text {coulomb }}\right|_{[0,1]^{n} \backslash S}$ obeys (44) and the Morrey space bounds. It remains to show that $\Delta A_{\text {coulomb }}$ $=\left.\Delta A_{\text {coulomb }}\right|_{[0,1]^{n} \backslash S}$ in the sense of distributions. Fortunately (42) will provide enough regularity on $A_{\text {coulomb }}$ to achieve this.

We turn to the details. Let $0<\delta \ll 1$ be a small parameter (which we will eventually send to zero). Since $S$ has Hausdorff dimension at most $n-4$, we can find a finite number of balls $B\left(x_{j}, r_{j}\right)$ with $0<r_{j}<\delta$ which cover $S$ and obey the bound

$$
\sum_{j} r_{j}^{n-3} \leq C \delta^{1 / 2}
$$

indeed, one could replace the exponent $1 / 2$ by any exponent between 0 and 1 .

For each ball $B\left(x_{j}, r_{j}\right)$, let $\eta_{j}$ be a non-negative bump function adapted to $B\left(x_{j}, 2 r_{j}\right)$ which equals 1 on $B\left(x_{j}, r_{j}\right)$, and define

$$
\eta:=\sup _{j} \eta_{j} .
$$

Thus $\eta$ is a Lipschitz function supported on $\bigcup_{j} B\left(x_{j}, 2 r_{j}\right)$ which equals 1 on $\bigcup_{j} B\left(x_{j}, r_{j}\right)$. We have the easily verified pointwise bound

$$
|\nabla \eta(x)| \leq C \sum_{j} r_{j}^{-1} \chi_{B\left(x_{j}, 2 r_{j}\right)} .
$$

From (42) we see that the function $(1-\eta) A_{\text {coulomb }}$ converges weakly to $A_{\text {coulomb }}$ as $\delta \rightarrow 0$. In particular, $\Delta\left((1-\eta) A_{\text {coulomb }}\right)$ converges weakly to $\Delta A_{\text {coulomb }}$ in the sense of distributions. On the other hand, $(1-\eta) \Delta A_{\text {coulomb }}$ is supported on $[0,1]^{n} \backslash S$ and so converges weakly to the $M_{4 / 3}^{3 n / 2}$ function $\left.\Delta A_{\text {coulomb }}\right|_{[0,1]^{n} \backslash S}$. To conclude the argument we have to show that the commutator estimate 


$$
\Delta\left((1-\eta) A_{\text {coulomb }}\right)-(1-\eta) \Delta A_{\text {coulomb }} \rightarrow 0
$$

as $\delta \rightarrow 0$.

The left-hand side of (47) is equal to

$$
-\nabla \eta \cdot \nabla A_{\text {coulomb }}-\nabla \cdot\left(\nabla \eta A_{\text {coulomb }}\right) .
$$

So it will suffice to show the strong $L^{1}$ convergence

$$
\left\|\nabla \eta \cdot \nabla A_{\text {coulomb }}\right\|_{L^{1}}+\left\|\nabla \eta A_{\text {coulomb }}\right\|_{L^{1}} \rightarrow 0 \text { as } \delta \rightarrow 0 .
$$

By (46) the left-hand side is bounded by

$$
C \sum_{j} r_{j}^{-1} \int_{B\left(x_{j}, 2 r_{j}\right)}\left|\nabla A_{\text {coulomb }}\right|+\left|A_{\text {coulomb }}\right| .
$$

By (42) and Hölder, we can bound this by

$$
C \sum_{j} r_{j}^{-1} \varepsilon r_{j}^{n-2}
$$

and the claim follows by (45).

We now use Lemma4.7 to show the interior decay estimate

$$
\left\|A_{\text {coulomb }}\right\|_{M_{2,1}^{n / 2}(B(x, \theta r))} \leq a\left\|A_{\text {coulomb }}\right\|_{M_{2,1}^{n / 2}(B(x, r))}
$$

for all balls $B(x, r) \subset(0,1)^{n}$, where $0<\theta \ll 1$ and $0<a<1$ are absolute constants to be chosen later.

By interior regularity (Lemma 3.7) we have

$$
\left\|A_{\text {coulomb }}\right\|_{M_{2,1}^{n / 2}(B(x, \theta r))} \leq C\left\|\Delta A_{\text {coulomb }}\right\|_{M_{4 / 3}^{3 n / 2}(B(x, r))}+C \theta^{2}\left\|A_{\text {coulomb }}\right\|_{M_{2,1}^{n / 2}(B(x, r))} \text {. }
$$

Applying (44) and using (14) we see that

$$
\begin{aligned}
\left\|A_{\text {coulomb }}\right\|_{M_{2,1}^{n / 2}(B(x, \theta r))} \leq & C\left(\left\|A_{\text {coulomb }}\right\|_{M_{2,1}^{n / 2}(B(x, r))}^{2}+\left\|A_{\text {coulomb }}\right\|_{M_{2,1}^{n / 2}(B(x, r))}^{3}\right) \\
& +C \theta^{2}\left\|A_{\text {coulomb }}\right\|_{M_{2,1}^{n / 2}(B(x, r))} .
\end{aligned}
$$

By (42) we thus have

$$
\left\|A_{\text {coulomb }}\right\|_{M_{2,1}^{n / 2}(B(x, \theta r))} \leq\left(C \theta^{2}+C \varepsilon\right)\left\|A_{\text {coulomb }}\right\|_{M_{2,1}^{n / 2}(B(x, r))},
$$

which gives the desired estimate (48) if $\theta$ and $\varepsilon$ are sufficiently small.

Iterating (48) we see that $A_{\text {coulomb }}$ is locally in $M_{2,1}^{n / 2+\delta}$ for some absolute constant $\delta>0$. Applying (14), (44), and elliptic estimates (such as variants of Lemma 3.7) we may bootstrap the value of $\delta$ in the usual fashion to obtain smoothness of $A_{\text {coulomb }}$ on $(0,1)^{n}$. We omit the details as they are rather standard.

The proof of Theorem 1.1 is thus complete as soon as we prove Proposition 4.3 Proposition 4.4, and Theorem 4.6. This will be achieved in Sections 574, Section 8 and Section 9. We remark that the proofs of these results are quite distinct and can be read independently of each other. 


\section{Integrating CONNECtions, CURVAture, AND AVERAGing ARGUMENTs}

We now begin the proof of Proposition 4.3 In this section we shall focus on developing the machinery needed to prove this proposition; more precisely, we set up some notation for integrating connections along paths and loops, and comparing the latter with integrals of curvature. We also need some machinery for averaging functions on the Lie group $G$ to obtain another function on $G$.

Throughout this section we assume that $A$ obeys the properties in Proposition 4.1, and in particular is smooth away from the set $S$.

If $x_{0}, x_{1}, x_{2} \in[0,1]^{n}$, we use $\Delta\left(x_{0}, x_{1}, x_{2}\right)$ to denote the solid triangle with these three vertices (or equivalently, the convex hull of $\left\{x_{0}, x_{1}, x_{2}\right\}$ ); this is a two-dimensional surface with boundary, and so we can integrate on it using twodimensional Hausdorff measure $d \mathbf{H}^{2}$.

If $x_{0}, x_{1} \in[0,1]^{n}$, we define $\left[x_{0} \rightarrow x_{1}\right]$ to be the path $t \mapsto x_{0}+t\left(x_{1}-x_{0}\right)$, i.e., the oriented line segment from $x_{0}$ to $x_{1}$. We use $\left[x_{0} \rightarrow x_{1} \rightarrow x_{2}\right]$ as shorthand for the concatenated path $\left[x_{0} \rightarrow x_{1}\right]+\left[x_{1} \rightarrow x_{2}\right]$; thus, for instance, the triangular loop $\left[x_{0} \rightarrow x_{1} \rightarrow x_{2} \rightarrow x_{0}\right]$ traverses the boundary of $\Delta\left(x_{0}, x_{1}, x_{2}\right)$.

Let $\left[x_{0} \rightarrow x_{1}\right]$ be a line segment which does not intersect the singular set $S$. We define the group element $A\left[\left[x_{0} \rightarrow x_{1}\right]\right] \in G$ by the PDE

$$
\begin{aligned}
A\left[\left[x_{0} \rightarrow x_{0}\right]\right] & =1_{G}, \\
\left(x_{1}-x_{0}\right) \cdot \nabla_{x_{1}} A\left[\left[x_{0} \rightarrow x_{1}\right]\right] & =A\left[\left[x_{0} \rightarrow x_{1}\right]\right]\left(\left(x_{1}-x_{0}\right) \cdot A\left(x_{1}\right)\right),
\end{aligned}
$$

where $1_{G}$ is the identity element of $G$.

Since $A$ is smooth on $\left[x_{0} \rightarrow x_{1}\right] \subset[0,1]^{n} \backslash S$, we see from the Picard existence theorem that $A\left[\left[x_{0} \rightarrow x_{1}\right]\right]$ is well-defined and takes values in $G$; indeed, this quantity is essentially the radial gauge from $x_{0}$ evaluated at $x_{1}$. In the special case where $G$ is abelian, we have the explicit formula

$$
\begin{aligned}
A\left[\left[x_{0} \rightarrow x_{1}\right]\right] & =\exp \left(\int_{\left[x_{0}, x_{1}\right]} A(y) d \mathbf{H}^{1}(y)\right) \\
& =\exp \left(\int_{0}^{1}\left(x_{1}-x_{0}\right) \cdot A\left(x_{0}+t\left(x_{1}-x_{0}\right)\right) d t\right)
\end{aligned}
$$

but in general no such explicit formula is available. Nevertheless, it is helpful to think of $A\left[\left[x_{0} \rightarrow x_{1}\right]\right]$ as some sort of non-abelian integral of $A$ from $x_{0}$ to $x_{1}$.

We define

$$
A\left[\left[x_{0} \rightarrow x_{1} \rightarrow x_{2}\right]\right]:=A\left[\left[x_{0} \rightarrow x_{1}\right]\right] A\left[\left[x_{1} \rightarrow x_{2}\right]\right]
$$

whenever $\left[x_{0} \rightarrow x_{1} \rightarrow x_{2}\right]$ does not intersect $S$, and similarly for more complicated polygonal paths. The expression $A\left[\left[x_{0} \rightarrow \ldots \rightarrow x_{n}\right]\right]$ can be regarded as the transport of the identity group element $1_{G}$ along the path $\left[x_{0} \rightarrow \ldots \rightarrow x_{n}\right]$ by the connection $A$; in particular, in the case of a loop $x_{n}=x_{0}$, this element represents the monodromy of the connection along the loop. In particular, for a small triangular loop we have

$$
A\left[\left[x_{0} \rightarrow x_{0}+\varepsilon v_{1} \rightarrow x_{0}+\varepsilon v_{2} \rightarrow x_{0}\right]\right]=1_{G}+\frac{\varepsilon^{2}}{2} F(A)\left(x_{0}\right)\left(v_{1}, v_{2}\right)+o\left(\varepsilon^{2}\right) ;
$$

indeed, this can be taken to be a more fundamental definition of the curvature $F(A)$ than (1). 
It is easy to verify the inversion law

$$
A\left[\left[x_{0} \rightarrow x_{1}\right]\right]=A\left[\left[x_{1} \rightarrow x_{0}\right]\right]^{-1}
$$

and the concatenation law

$$
A\left[\left[x_{0} \rightarrow x_{1} \rightarrow x_{2}\right]\right]=A\left[\left[x_{0} \rightarrow x_{2}\right]\right]
$$

when $x_{1}$ lies in $\left[x_{0} \rightarrow x_{2}\right]$; these allow us to perform manipulations such as

$$
A\left[\left[x_{0} \rightarrow x_{1} \rightarrow x_{2} \rightarrow x_{0}\right]\right] A\left[\left[x_{0} \rightarrow x_{2} \rightarrow x_{3} \rightarrow x_{0}\right]\right]=A\left[\left[x_{0} \rightarrow x_{1} \rightarrow x_{3} \rightarrow x_{0}\right]\right]
$$

whenever $x_{2}$ lies in $\left[x_{1} \rightarrow x_{3}\right]$.

By conjugating (49) by $\sigma$ it is easy to arrive at the gauge transformation law

$$
\sigma(A)\left[\left[x_{0} \rightarrow x_{1}\right]\right]=\sigma\left(x_{0}\right) A\left[\left[x_{0} \rightarrow x_{1}\right]\right] \sigma\left(x_{1}\right)^{-1}
$$

whenever $\left[x_{0} \rightarrow x_{1}\right]$ is disjoint from $S$. More generally we have

$$
\sigma(A)\left[\left[x_{0} \rightarrow x_{1} \rightarrow x_{2}\right]\right]=\sigma\left(x_{0}\right) A\left[\left[x_{0} \rightarrow x_{1} \rightarrow x_{2}\right]\right] \sigma\left(x_{2}\right)^{-1},
$$

whenever $\left[x_{0} \rightarrow x_{1} \rightarrow x_{2}\right]$ is disjoint from $S$, etc.

If $A$ has no curvature, $F(A)=0$, then the monodromy along any loop is zero, and in particular we have $A\left[\left[x_{0} \rightarrow x_{1} \rightarrow x_{2} \rightarrow x_{0}\right]\right]=1_{G}$ whenever the triangle $\Delta\left(x_{0}, x_{1}, x_{2}\right)$ does not intersect $S$. When the curvature is non-zero, we can still estimate the monodromy by the integral of the curvature:

Lemma 5.1 (Non-abelian Stokes theorem). Let $x_{0}, x_{1}, x_{2} \in[0,1]^{n}$ be such that the triangle $\Delta\left(x_{0}, x_{1}, x_{2}\right)$ is disjoint from $S$. Then

$$
\left|A\left[\left[x_{0} \rightarrow x_{1} \rightarrow x_{2} \rightarrow x_{0}\right]\right]-1_{G}\right| \leq C \int_{\Delta\left(x_{0}, x_{1}, x_{2}\right)}|F(A)(y)| d \boldsymbol{H}^{2}(y)
$$

where $d \boldsymbol{H}^{2}(y)$ is two-dimensional Hausdorff measure.

Proof. It will be convenient to replace $\left|A[]-1_{G}\right|$ by a slightly different quantity. Let $d($, ) be the arclength metric on the group $G \subseteq U(N)$; this metric is bi-invariant under left and right multiplication by elements of $G$, and $d\left(g, g^{\prime}\right) \sim\left|g-g^{\prime}\right|$. Thus it will suffice to prove the estimate

$$
d\left(A\left[\left[x_{0} \rightarrow x_{1} \rightarrow x_{2} \rightarrow x_{0}\right]\right], 1_{G}\right) \leq C \int_{\Delta\left(x_{0}, x_{1}, x_{2}\right)}|F(A)(y)| d \mathbf{H}^{2}(y) .
$$

The claim is invariant under cyclic permutation of $x_{0}, x_{1}, x_{2}$. Also, if we let $x_{3}$ be the midpoint of $x_{0}$ and $x_{2}$, then from the invariance properties of the metric and the triangle inequality we have

$$
\begin{aligned}
d\left(A\left[\left[x_{0} \rightarrow x_{1} \rightarrow x_{2} \rightarrow x_{0}\right]\right], 1_{G}\right) \leq & d\left(A\left[\left[x_{0} \rightarrow x_{1} \rightarrow x_{3} \rightarrow x_{0}\right]\right], 1_{G}\right) \\
& +d\left(A\left[\left[x_{3} \rightarrow x_{2} \rightarrow x_{1} \rightarrow x_{3}\right]\right], 1_{G}\right)
\end{aligned}
$$

while we trivially have

$$
\begin{aligned}
\int_{\Delta\left(x_{0}, x_{1}, x_{2}\right)}|F(A)(y)| d \mathbf{H}^{2}(y)= & \int_{\Delta\left(x_{0}, x_{1}, x_{3}\right)}|F(A)(y)| d \mathbf{H}^{2}(y) \\
& +\int_{\Delta\left(x_{3}, x_{2}, x_{1}\right)}|F(A)(y)| d \mathbf{H}^{2}(y) .
\end{aligned}
$$

Thus to prove the claim for the triangle $\Delta\left(x_{0}, x_{1}, x_{2}\right)$ it suffices to do so for the two smaller triangles $\Delta\left(x_{0}, x_{1}, x_{3}\right)$ and $\Delta\left(x_{3}, x_{2}, x_{1}\right)$ (with exactly the same constant 
$C)$. Repeating this calculation indefinitely we thus see that it will suffice to prove this estimate for infinitesimal triangles. But this follows directly from (50).

Of course, all the above results only hold subject to the caveat that various lines and triangles do not intersect $S$. Fortunately, if one has enough free parameters, then these intersections are extremely rare 8

Lemma 5.2. For any point $x_{0} \notin S$, the set $\left\{x \in \boldsymbol{R}^{n}:\left[x_{0} \rightarrow x\right]\right.$ intersects $\left.S\right\}$ has measure zero. For any $x_{0}, x_{1} \notin S$ with $\left[x_{0} \rightarrow x_{1}\right]$ not intersecting $S$, the set $\left\{x \in \boldsymbol{R}^{n}: \Delta\left(x_{0}, x_{1}, x\right)\right.$ intersects $\left.S\right\}$ has measure zero.

Proof. It suffices to prove the second claim, since the first follows by setting $x_{0}=x_{1}$.

Fix $x_{0}, x_{1}$. If $\Delta\left(x_{0}, x_{1}, x\right)$ intersects $S$, then we must have

$$
y=(1-\alpha)\left((1-\theta) x_{0}+\theta x_{1}\right)+\alpha x
$$

for some $0 \leq \alpha, \theta \leq 1$ and $y \in S$. Since $\left[x_{0} \rightarrow x_{1}\right]$ is disjoint from the compact set $S$, we see that $\alpha>c>0$ for some $c=c\left(x_{0}, x_{1}, S\right)$. We now solve for $x$ as

$$
x=\frac{1}{\alpha} y-\frac{1-\alpha}{\alpha}\left((1-\theta) x_{0}+\theta x_{1}\right) .
$$

Since $y$ lives in a set of dimension at most $n-4$, and $\alpha$ and $\theta$ are one-dimensional parameters with $1 / \alpha$ bounded, it is easy to see that $x$ lives in a set of dimension at most $n-2$, which necessarily has measure zero, as desired.

To exploit this generic lack of intersections, we shall use an averaging argument, using a random origin $x_{0}$ to create a partially defined gauge (e.g., by using a radial gauge $\sigma(x)=A\left[\left[x_{0} \rightarrow x\right]\right]$, defined as long as $\left[x_{0} \rightarrow x\right]$ does not intersect $\left.S\right)$, and then averaging over $x_{0}$ (using Lemma [5.2) to recover a globally defined gauge.

To do this we need a notion of averaging 9 on the Lie group $G$. Suppose that we have a domain $\Omega \subseteq[0,1]^{n}$, a weight function $a: \Omega \rightarrow \mathbf{R}^{+}$with $0<\|a\|_{L^{1}(\Omega)}<\infty$, and a measurable map $f: \Omega \rightarrow G$ defined for a.e. $x \in \Omega$. We would like to define a group element $[f]_{\Omega, a}^{G} \in G$, which represents in some sense an "average" of $f(x)$ where $x$ ranges over the probability measure $a d x /\|a\|_{L^{1}(\Omega)}$.

Since $G$ is embedded in the vector space $M_{N}(\mathbf{C})$ of $N \times N$ complex matrices, we can define the linear average $[f]_{\Omega, a} \in M_{N}(\mathbf{C})$ by

$$
[f]_{\Omega, a}:=\frac{\int_{\Omega} f(x) a(x) d x}{\int_{\Omega} a(x) d x},
$$

but of course this average will almost certainly lie outside of the group $G$. To resolve this problem, we observe from the compactness of $G$ that there exists a tubular $\delta$-neighbourhood $N_{\delta}(G)$ of $G$ for some fixed $0<\delta \ll 1$ for which there is a smooth projection map $\pi: N_{\delta}(G) \rightarrow G$ which equals the identity on $G$. We can in fact choose $\pi$ to obey the right-equivariance condition $\pi(x g)=\pi(x) g$ for all

\footnotetext{
${ }^{8} \mathrm{~A}$ variant of this argument can be used to show that $[0,1]^{n} \backslash S$ is simply connected; indeed, any closed loop in $[0,1]^{n} \backslash S$ can be contracted along a generic cone over that loop. While we will not use this fact directly, it does shed some light as to why gauge transform results such as Proposition 4.3 or Proposition 4.4 are possible.

${ }^{9}$ One can view the machinery here as a continuous version of the more discrete gauge gluing techniques in, say, [6]. We were forced to use this continuous procedure instead of the discrete one in order to preserve the constants in the inductive procedure in Section 7
} 
$x \in N_{\delta}(G)$ and $g \in G$. In particular we observe (since $\pi$ is Lipschitz) that we have the estimate

$$
\left|\pi(x) g \pi(y)^{-1}-1\right|=\left|\pi(x g) \pi(y)^{-1}-1\right| \leq C|\pi(x g)-\pi(y)| \leq C|x g-y|
$$

for all $g \in G$ and $x, y \in N_{\delta}(G)$.

We now define

$$
[f]_{\Omega, a}^{G}:=\pi\left([f]_{\Omega, a}\right)
$$

provided that the average $[f]_{\Omega, a}$ lies in $N_{\delta}(G)$. This can be achieved provided that the values of $f$ "cluster together"; more precisely, we have

Lemma 5.3. Let the notation and assumptions be as above. If $f$ obeys the clustering condition

$$
\int_{\Omega} \int_{\Omega}|f(x)-f(y)| a(x) a(y) d \mu(x) d \mu(y)<\delta\|a\|_{L^{1}(\Omega)}^{2},
$$

then $[f]_{\Omega, a}$ lies in $N_{\delta}(G)$, and so $[f]_{\Omega, a}^{G}$ is well-defined. Here we use $d \mu$ to denote Haar measure on the compact group $G$, normalized so that $\mu(G)=1$.

Proof. We may of course assume that $f(x)$ is defined and takes values in $G$ for all $x \in X$, since sets of measure zero are clearly irrelevant here.

By (56) and the pigeonhole principle we may find an $x \in X$ such that

$$
\int_{\Omega}|f(x)-f(y)| a(y) d \mu(y)<\delta\|a\|_{L^{1}(\Omega)} .
$$

From the triangle inequality we thus have

$$
\left|\int_{\Omega}(f(x)-f(y)) a(y) d \mu(y)\right|<\delta\|a\|_{L^{1}(\Omega)} .
$$

But the left-hand side simplifies to

$$
\left|f(x)-[f]_{\Omega, a}\right|\|a\|_{L^{1}(\Omega)} .
$$

Thus $[f]_{\Omega, a}$ lies within distance $\delta$ of the point $f(x) \in G$, and the claim follows.

\section{Fractional integration bounds}

In the next section we shall be constructing gauges by averaging certain integrals of the form $A\left[\left[x_{0} \rightarrow x_{1} \rightarrow x_{2}\right]\right]$ using Lemma [5.3, We will then use Lemma 5.1 to estimate the expressions which then result. This will lead to integrating the curvature on an "averaged collection of 2-surfaces"; to assist the proof of Proposition 4.3 we now present a simple lemma (mostly a consequence of the change of variables formula) to understand such integrals.

Lemma 6.1. For any $0<r \leq R \leq 1$ and $x \in[0,1]^{n}$, we have

$$
\begin{aligned}
\int_{B(x, R)} & \int_{B(x, r)} \int_{\Delta\left(x, x_{1}, x_{2}\right)}|F(A)(y)| d \boldsymbol{H}^{2}(y) d x_{1} d x_{2} \\
\leq & C r^{n} R^{n}\left(\int_{B(x, r)}|F(A)(y)||x-y|^{2-n} d y\right. \\
& \left.+r \int_{B(x, 2 R) \backslash B(x, r)}|F(A)(y)||x-y|^{1-n} d y\right) .
\end{aligned}
$$


Proof. We parameterize $x_{1}=x+r z, x_{2}=x+R z^{\prime}$ and $y=x+\operatorname{tr} z+t^{\prime} R z^{\prime}$ for $z, z^{\prime} \in B(0,1)$ and $t, t^{\prime} \in[0,1]$ (we also have $t+t^{\prime} \leq 1$, but we will not need this), and bound the left-hand side of (57) by

$$
C r^{n} R^{n} r R \int_{B(0,1)} \int_{B(0,1)} \int_{0}^{1} \int_{0}^{1}\left|F(A)\left(x+t r z+t^{\prime} R z^{\prime}\right)\right| d t d t^{\prime} d z d z^{\prime} .
$$

Making the change of variables $\left(t, t^{\prime}, w, w^{\prime}\right)=\left(t, t^{\prime}, t z, t z^{\prime}\right)$, this becomes

$$
C r^{n} R^{n} r R \int_{0}^{1} \int_{0}^{1} \int_{B\left(0, t^{\prime}\right)} \int_{B(0, t)}\left(t t^{\prime}\right)^{n}\left|F(A)\left(x+r w+R w^{\prime}\right)\right| d w d w^{\prime} d t d t^{\prime} .
$$

Applying Fubini's theorem and performing the $t, t^{\prime}$ integrals, this becomes

$$
C r^{n} R^{n} r R \int_{B(0,1)} \int_{B(0,1)}|w|^{1-n}\left|w^{\prime}\right|^{1-n}\left|F(A)\left(x+r w+R w^{\prime}\right)\right| d w d w^{\prime} .
$$

Making the change of variables $(w, y)=\left(w, x+r w+R w^{\prime}\right)$, and noting that $y \in$ $B(x, 2 R)$, this can be bounded by

$$
C r^{n} R^{n} r R R^{-n} \int_{B(x, 2 R)} \int_{B(0,1)}\left(\frac{|y-x-r w|}{R}\right)^{1-n}|w|^{1-n}|F(A)(y)| d w d y .
$$

Comparing this with (57), it thus suffices to show that

$$
\int_{B(0,1)}|y-x-r w|^{1-n}|w|^{1-n} d w \leq C|y-x|^{2-n} / r
$$

when $|y-x| \leq r$ and

$$
\int_{B(0,1)}|y-x-r w|^{1-n}|w|^{1-n} d w \leq C|y-x|^{1-n}
$$

otherwise. But this can be verified by a direct computation.

From the above lemma we see that it is of interest to compute various fractional integrals of $F(A)$; we record two such computations below.

Lemma 6.2. If $x \in \Omega_{m}$ and $r>0$, then

$$
\int_{B(x, r)}|F(A)(y)||x-y|^{2-n} d y \leq C \varepsilon \log \left(2+\frac{r}{R_{m}}\right) .
$$

We also have the variant estimate: if $x \in \Omega_{m+1}$, then

$$
\int_{[0,1]^{n}} \min \left(1,\left(\frac{R_{m}}{|x-y|}\right)^{1-\kappa / 2}\right) \frac{|F(A)(y)|}{|x-y|^{n-2}} d y \leq C \varepsilon \log D .
$$

Proof. We decompose the left-hand side of (58) dyadically and bound it by

$$
C \sum_{j=0}^{\infty}\left(2^{-j} r\right)^{2-n} \int_{B\left(x, 2^{-j} r\right)}|F(A)| .
$$

By (29) we have the bound

$$
\int_{B\left(x, 2^{-j} r\right)}|F(A)| \leq C \varepsilon\left(2^{-j} r\right)^{n-2} .
$$


On the other hand, since $x \in \Omega_{m}$, we have $Q(x) \leq \varepsilon D^{(1-\kappa) m}=C \varepsilon R_{m}^{-1+\kappa}$, which implies from (31) and Hölder that

$$
\int_{B\left(x, 2^{-j} r\right)}|F(A)| \leq C\left(2^{-j} r\right)^{n / 2}\left(\int_{B\left(x, 2^{-j} r\right)}|F(A)|^{2}\right)^{1 / 2} \leq C \varepsilon\left(2^{-j} r\right)^{n-1-\kappa} R_{m}^{-1+\kappa} .
$$

Thus we can estimate the left-hand side of (60) by

$$
C \sum_{j=0}^{\infty} \min \left(\varepsilon, \varepsilon\left(2^{-j} r / R_{m}\right)^{1-\kappa}\right)
$$

and the claim (58) follows.

Now we prove (59). The portion of the integral when $|x-y| \leq R_{m}$ is acceptable by (58) (with $m$ replaced by $m+1$, and recalling that $R_{m} / R_{m+1}=D$ ). The portion when $|x-y|>R_{m}$ can be decomposed dyadically and estimated by

$$
C \sum_{j=0}^{\infty} 2^{-j(1-\kappa / 2)}\left(2^{j} R_{m}\right)^{2-n} \int_{B\left(x, 2^{j} R_{m}\right)}|F(A)| .
$$

By (29) this can be bounded by

$$
C \sum_{j=0}^{\infty} 2^{-j(1-\kappa / 2)} \varepsilon,
$$

which is acceptable.

From the above lemma we see that the integral

$$
\int_{[0,1]^{n}} \frac{|F(A)(y)|}{|x-y|^{n-2}} d y
$$

might diverge logarithmically, like $\varepsilon|\log \rho(x)|$, as one approaches the singular set $S$. This is rather unfortunate; if this integral were uniformly bounded by $O(\varepsilon)$, then one would not need the rather complicated inductive argument below, as the $m=1$ iteration of the gauge would already extend all the way down to $S$. It is interesting that Price's monotonicity formula 2] does give some additional control on the radial component $F(A)(y) \cdot \frac{x-y}{|x-y|}$ of the curvature, in particular obtaining an estimate of the form

$$
\int_{[0,1]^{n}} \frac{\left|F(A)(y) \cdot \frac{x-y}{\mid x-y}\right|^{2}}{|x-y|^{n-4}} d y \leq C \varepsilon .
$$

Furthermore, in all the arguments in the next section it turns out that we only need the radial component of the curvature. Unfortunately, this bound only seems able to improve the logarithmic divergence slightly, to $O(\varepsilon \sqrt{|\log \rho(x)|})$, but cannot eliminate it entirely. Thus we have been forced to perform this somewhat artificial and complicated inductive procedure in order to obtain a gauge which extends arbitrarily close to the singular set $S$ and which obeys manageable bounds on the connection $\sigma_{m}(A)$. 


\section{Proof of Proposition 4.3}

Armed with all the above machinery we can now prove Proposition 4.3

We proceed by induction on $m$. For inductive purposes we shall need to make certain constants explicit; specifically, we shall need a large constant $C_{1}$ depending on $D, n$, and $G$. The precise inductive claim is as follows:

Proposition 7.1. For each $m \geq 1$, there exists a gauge $\sigma_{m}$ with the following Lipschitz property: whenever $x_{0}, x_{1} \in \Omega_{m}$ and $r>0$ are such that $\left|x_{0}-x_{1}\right| \leq r \leq$ $10 R_{m}$ we hav 10

$$
\begin{gathered}
r^{-n} \int_{B\left(x_{0}, r\right) \cap[0,1]^{n}}\left|\sigma_{m}\left(x_{0}\right) A\left[\left[x_{0} \rightarrow x_{2} \rightarrow x_{1}\right]\right] \sigma_{m}\left(x_{1}\right)^{-1}-1_{G}\right| d x_{2} \\
\leq C_{1} r\left(T_{m}\left(x_{0}\right)+T_{m}\left(x_{1}\right)\right) .
\end{gathered}
$$

Suppose for the moment that Proposition 7.1 held. Let $x_{0} \in \Omega_{m}$. If we set $x_{1}=x_{0}+\epsilon v$ and $r:=\epsilon$ for some unit vector $v$ and some $\epsilon$ small enough that $B\left(x_{0}, 2 \epsilon\right) \subseteq \Omega_{m}$, then from (53) we have

$$
\left|\sigma_{m}\left(x_{0}\right) A\left[\left[x_{0} \rightarrow x_{2} \rightarrow x_{1}\right]\right] \sigma_{m}\left(x_{1}\right)^{-1}-1\right|=\left|\sigma_{m}(A)\left[\left[x_{0} \rightarrow x_{2} \rightarrow x_{1}\right]\right]-1\right|
$$

whenever $x_{2} \in B\left(x_{0}, r\right) \cap[0,1]^{n}$, which by (49) implies that

$$
\left|\sigma_{m}\left(x_{0}\right) A\left[\left[x_{0} \rightarrow x_{2} \rightarrow x_{1}\right]\right] \sigma_{m}\left(x_{1}\right)^{-1}-1\right| \geq C^{-1} \epsilon\left|v \cdot \sigma_{m}(A)\left(x_{0}\right)\right|+o(\epsilon) .
$$

Combining this with (53) and (61), we obtain

$$
\epsilon\left|v \cdot \sigma_{m}(A)\left(x_{0}\right)\right|+o(\epsilon) \leq C_{1} \epsilon\left(T_{m}\left(x_{0}\right)+T_{m}\left(x_{0}+\epsilon v\right)\right) .
$$

Dividing by $\epsilon$ and then taking limits as $\epsilon \rightarrow 0$, we obtain (36) as desired, since $v$ was arbitrary (note that $T_{m}$ is continuous on $[0,1]^{n} \backslash S$, and in particular on $\Omega_{m}$ ). Thus to prove Proposition 4.3 it will suffice to prove Proposition 7.1 As with all inductions, this is done in two steps.

Step 1. The base case $m=1$.

We first construct the initial gauge $\sigma_{1}$. We remark that it would be relatively easy to start the induction if we knew that $[0,1]^{n} \backslash S$ contained a large ball, but we are making no assumptions on $S$ other than the dimension assumption and so cannot assume this. Besides, the arguments we will use here will also motivate the inductive step below.

We first pick a good choice of origin $x_{*} \in \Omega_{1}$. From Fubini's theorem we have

$$
\int_{\Omega_{1}} \int_{[0,1]^{n}} \frac{|F(A)(x)|}{\left|x-x_{*}\right|^{n-2}} d x d x_{*} \leq C \int_{[0,1]^{n}}|F(A)(x)| d x .
$$

From (35) and the pigeonhole principle, there thus exists $x_{*} \in \Omega_{1}$ such that

$$
\int_{[0,1]^{n}} \frac{|F(A)(x)|}{\left|x-x_{*}\right|^{n-2}} d x \leq C \int_{[0,1]^{n}}|F(A)(x)| d x .
$$

Fix this $x_{*}$. For each $x_{1} \in[0,1]^{n}$ we define the group element $f_{x, 0}\left(x_{1}\right) \in G$ by

$$
f_{x, 0}\left(x_{1}\right):=A\left[\left[x_{*} \rightarrow x_{1} \rightarrow x\right]\right] .
$$

This group element is undefined if $\left[x_{*} \rightarrow x_{1} \rightarrow x\right]$ intersects $S$, but from Lemma 5.2 we see that the set of $x_{1}$ for which that occurs has measure zero. Thus $f_{x, 0}$ :

\footnotetext{
${ }^{10}$ Of course, the integrand is only defined if $\left[x_{0} \rightarrow x_{2} \rightarrow x_{1}\right]$ avoids $S$, but this will turn out to be the case for almost every $x_{2}$. See Lemma 5.2
} 
$[0,1]^{n} \rightarrow G$ is defined at almost every point of $[0,1]^{n}$, and there will be no difficulty integrating this function in the $x_{1}$ variable.

Lemma 7.2 (Clustering bound for $\sigma_{1}$ ). For all $x \in \Omega_{1}$, we have the clustering bound

(64)

$$
\int_{[0,1]^{n}} \int_{[0,1]^{n}}\left|f_{x, 0}\left(x_{1}\right)-f_{x, 0}\left(x_{2}\right)\right| d x_{1} d x_{2} \leq C \int_{[0,1]^{n}}|F(A)(y)||x-y|^{2-n} d y .
$$

Proof. From (63), (51) we see that $f_{x_{0}}\left(x_{1}\right)-f_{x_{0}}\left(x_{2}\right)$ is conjugate to $A\left[\left[x_{*} \rightarrow x_{1} \rightarrow\right.\right.$ $\left.\left.x \rightarrow x_{2} \rightarrow x_{*}\right]\right]-1_{G}$, and in particular that

$$
\left|f_{x, 0}\left(x_{1}\right)-f_{x, 0}\left(x_{2}\right)\right|=\left|A\left[\left[x_{*} \rightarrow x_{1} \rightarrow x \rightarrow x_{2} \rightarrow x_{*}\right]\right]-1_{G}\right| .
$$

The loop on the right-hand side is the boundary of $\Delta\left(x_{*}, x_{1}, x_{2}\right) \cup \Delta\left(x, x_{1}, x_{2}\right)$, where $\Delta\left(x, x_{1}, x_{2}\right)$ denotes the triangle with vertices $x, x_{1}, x_{2}$. For almost every $x_{1}$, we see from Lemma 5.2 that the triangles $\Delta\left(x_{*}, x_{1}, x_{2}\right)$ and $\Delta\left(x, x_{1}, x_{2}\right)$ are disjoint from $S$ for almost every $x_{2}$. We may thus apply Lemma 5.1. and bound the left-hand side of (64) by

$$
C \int_{[0,1]^{n}} \int_{[0,1]^{n}}\left(\int_{\Delta\left(x, x_{1}, x_{2}\right) \cup \Delta\left(x_{*}, x_{1}, x_{2}\right)}|F(A)(y)| d \mathbf{H}^{2}(y)\right) d x_{1} d x_{2} .
$$

By Lemma 6.1 we can bound this by

$$
C \int_{[0,1]^{n}}|F(A)(y)|\left(|x-y|^{2-n}+\left|x_{*}-y\right|^{2-n}\right) d y .
$$

The claim then follows from (62).

From (64) and Lemma 6.2 we have in particular that

$$
\int_{[0,1]^{n}} \int_{[0,1]^{n}}\left|f_{x, 0}\left(x_{1}\right)-f_{x, 0}\left(x_{2}\right)\right| d x_{1} d x_{2} \leq C \varepsilon \log D
$$

for $x \in \Omega_{1}$.

We now define $\sigma_{1}$ by averaging $f_{x, 0}$ on the cube $[0,1]^{n}$ :

$$
\sigma_{1}(x):=\left[f_{x, 0}\right]_{[0,1]^{n}, 1}^{G}=\pi\left(\left[f_{x, 0}\right]_{[0,1]^{n}, 1}\right) ;
$$

from (65) and Lemma 5.3 we see that $\sigma_{1}$ is well-defined on $\Omega_{1}$, if $\varepsilon$ is sufficiently small depending on $D$. We now verify the condition (61); in other words, we show that whenever $x_{0}, x_{1} \in \Omega_{1}$ and $\left|x_{0}-x_{1}\right| \leq r \leq 10 R_{1}$, we have the bound

$$
\begin{aligned}
r^{-n} \int_{B\left(x_{0}, r\right) \cap[0,1]^{n}} \mid \pi\left(\left[f_{x_{0}, 0}\right]_{[0,1]^{n}, 1}\right) A\left[\left[x_{0} \rightarrow x_{2} \rightarrow x_{1}\right]\right] & \pi\left(\left[f_{x_{1}, 0}\right]_{[0,1]^{n}, 1}\right)^{-1}-1_{G} \mid d x_{2} \\
\leq & C_{1} r\left(T_{1}\left(x_{0}\right)+T_{1}\left(x_{1}\right)\right) .
\end{aligned}
$$

Fix $x_{0}, x_{1}, r$. By (55), we can bound the left-hand side of (66) by

$$
C r^{-n} \int_{B\left(x_{0}, r\right) \cap[0,1]^{n}}\left|\left[f_{x_{0}, 0}\right]_{[0,1]^{n}, 1} A\left[\left[x_{0} \rightarrow x_{2} \rightarrow x_{1}\right]\right]-\left[f_{x_{1}, 0}\right]_{[0,1]^{n}, 1}\right| ;
$$

by the triangle inequality, this is bounded by

$$
C r^{-n} \int_{B\left(x_{0}, r\right) \cap[0,1]^{n}} \int_{[0,1]^{n}}\left|f_{x_{0}, 0}(x) A\left[\left[x_{0} \rightarrow x_{2} \rightarrow x_{1}\right]\right]-f_{x_{1}, 0}(x)\right| d x d x_{2} .
$$


From (63), (51) we see that $f_{x_{0}, 0}(x) A\left[\left[x_{0} \rightarrow x_{2} \rightarrow x_{1}\right]\right]-f_{x_{1}, 0}(x)$ is conjugate to $A\left[\left[x \rightarrow x_{0} \rightarrow x_{2} \rightarrow x_{1} \rightarrow x\right]\right]-1_{G}$; thus, we can rewrite the previous as

$$
C r^{-n} \int_{B\left(x_{0}, r\right) \cap[0,1]^{n}} \int_{[0,1]^{n}}\left|A\left[\left[x \rightarrow x_{0} \rightarrow x_{2} \rightarrow x_{1} \rightarrow x\right]\right]-1_{G}\right| d x d x_{2} .
$$

Applying Lemma 5.1 we can bound this integral by

$$
C r^{-n} \int_{B\left(x_{0}, r\right) \cap[0,1]^{n}} \int_{[0,1]^{n}}\left(\int_{\Delta\left(x, x_{2}, x_{0}\right) \cup \Delta\left(x, x_{2}, x_{1}\right)}|F(A)(y)| d \mathbf{H}^{2}(y)\right) d x d x_{2} .
$$

First consider the integral on $\Delta\left(x, x_{2}, x_{0}\right)$. From Lemma 6.1 (and using the crude estimate $|x-y|^{2-n} \leq r|x-y|^{1-n}$ when $\left.|x-y| \leq r\right)$ we can bound this by

$$
C r \int_{[0,1]^{n}}\left|x_{0}-y\right|^{n-1}|F(A)(y)| d y \leq C(D) r T_{1}\left(x_{0}\right)
$$

as desired. The contribution of the triangles $\Delta\left(x, x_{2}, x_{1}\right)$ is similar, but with $T_{1}\left(x_{0}\right)$ replaced by $T_{1}\left(x_{1}\right)$. This proves 66 .

This completes the proof of the base case $m=1$.

Step 2. Induct from $m$ to $m+1$.

We now assume that Proposition 7.1 has already been proven for $m$, and now construct a gauge $\sigma_{m+1}$ on $\Omega_{m+1}$ with the desired properties. This will basically be the same argument as Step 1 , but rescaled by a factor of $D^{-m}$ and with some minor technical changes. The key point here is that when moving from $\sigma_{m}$ to $\sigma_{m+1}$, the bound on the right-hand side of (61) worsens by a factor of about $D$, which will allow us to close the argument if $D$ is sufficiently large.

Let $x \in \Omega_{m+1}$, and consider the ball $B_{x}:=B\left(x, R_{m}\right)$. Let $\psi_{x}$ be the cutoff function $\psi_{x}(y):=\psi\left((y-x) / R_{m}\right)$, where $\psi$ is a non-negative bump function adapted to $B(0,2)$ which equals one on $B(0,1)$; thus $\psi_{x}$ equals one on $B_{x}$. In particular, from (35) we have

$$
\left\|\psi_{x}\right\|_{L^{1}\left(\Omega_{m}\right)} \geq\left|B_{x} \cap \Omega_{m}\right| \geq C^{-1} R_{m}^{n}
$$

We define the function $f_{x, m}$ on $\Omega_{m}$ by

$$
f_{x, m}\left(x_{1}\right):=\sigma_{m}\left(x_{1}\right) A\left[\left[x_{1} \rightarrow x\right]\right] ;
$$

observe that Lemma 5.2 ensures that $f_{x}$ is defined almost everywhere on $\Omega_{m}$.

One of the key observations we will need is that if we vary $x$ by $O\left(R_{m+1}\right)$, then $\psi_{x}$ only varies by at most $O\left(R_{m+1} / R_{m}\right)=O(1 / D)$; this $1 / D$ gain will be crucial in allowing us to close the induction. This is the main reason why we need a large dyadic base $D$ instead of just using the standard powers of two.

The analogue of Lemma 7.2 is

Lemma 7.3 (Clustering bound for $\sigma_{m}$ ). For all $x \in \Omega_{m+1}$, we have the clustering bound

$$
\begin{aligned}
& \int_{B\left(x, 5 R_{m}\right)} \int_{B\left(x, 5 R_{m}\right)}\left|f_{x, m}\left(x_{1}\right)-f_{x, m}\left(x_{2}\right)\right| d x_{1} d x_{2} \\
& \leq C C_{1} R_{m}^{2 n} \int_{[0,1]^{n}} \min \left(1,\left(\frac{R_{m}}{|x-y|}\right)^{1-\kappa / 2}\right) \frac{|F(A)(y)|}{|x-y|^{n-2}} d y .
\end{aligned}
$$


Proof. Let $x_{1}, x_{2} \in B\left(x, 5 R_{m}\right)$. By (69), (51) we have

$$
\left|f_{x, m}\left(x_{1}\right)-f_{x, m}\left(x_{2}\right)\right|=\left|\sigma_{m}\left(x_{1}\right) A\left[\left[x_{1} \rightarrow x \rightarrow x_{2}\right]\right] \sigma_{m}\left(x_{2}\right)^{-1}-1_{G}\right|
$$

since the expressions inside the absolute values are conjugate. We insert a dummy variable $x_{3}$, ranging over $B\left(x_{1}, 20 R_{m}\right) \cap[0,1]^{n}$, and average to obtain

$$
\begin{aligned}
& \left|f_{x, m}\left(x_{1}\right)-f_{x, m}\left(x_{2}\right)\right| \\
& \quad \leq C R_{m}^{-n} \int_{B\left(x_{1}, 20 R_{m}\right) \cap[0,1]^{n}}\left|\sigma_{m}\left(x_{1}\right) A\left[\left[x_{1} \rightarrow x \rightarrow x_{2}\right]\right] \sigma_{m}\left(x_{2}\right)^{-1}-1_{G}\right| d x_{3} .
\end{aligned}
$$

We use the groupoid properties again and the triangle inequality to estimate the right-hand side by the sum of

$$
C R_{m}^{-n} \int_{B\left(x_{1}, 20 R_{m}\right) \cap[0,1]^{n}}\left|\sigma_{m}\left(x_{1}\right) A\left[\left[x_{1} \rightarrow x_{3} \rightarrow x_{2}\right]\right] \sigma_{m}\left(x_{2}\right)^{-1}-1_{G}\right| d x_{3}
$$

and

$$
C R_{m}^{-n} \int_{B\left(x_{1}, 20 R_{m}\right) \cap[0,1]^{n}}\left|A\left[\left[x_{1} \rightarrow x_{3} \rightarrow x_{2} \rightarrow x \rightarrow x_{1}\right]\right]-1_{G}\right| d x_{3} .
$$

Consider first the contribution of (71) to (70). Since $x_{1}, x_{2} \in B\left(x, 5 R_{m}\right)$, we have $\left|x_{1}-x_{2}\right| \leq 10 R_{m}$. Thus the inductive hypothesis (61) applies, and we have the bound

$$
\text { (71) } \leq C C_{1} R_{m}\left(T_{m}\left(x_{1}\right)+T_{m}\left(x_{2}\right)\right) \text {. }
$$

By symmetry, the contribution of (71) to the left-hand side of (70) can thus be estimated by

$$
C C_{1} R_{m}^{n} R_{m} \int_{B\left(x, 2 R_{m}\right)} T_{m}(y) d y,
$$

which in turn can be estimated using (37) and Fubini's theorem by

$$
C C_{1} R_{m}^{n} R_{m} \int_{[0,1]^{n}} \min \left(R_{m}, R_{m}^{n-\kappa / 2}|x-y|^{1-n+\kappa / 2}\right)|F(A)(y)| d y,
$$

which is acceptable.

Now we consider the contribution of (72). By Lemma 5.2 we see that the triangles $\Delta\left(x, x_{1}, x_{3}\right)$ and $\Delta\left(x_{3}, x_{2}, x\right)$ will not intersect $S$ for almost every choice of $x_{1}, x_{2}, x_{3}$. Thus we may use Lemma 5.1 and bound the contribution to (70) by

$$
\begin{aligned}
C R_{m}^{-n} & \int_{B\left(x, 5 R_{m}\right)} \int_{B\left(x, 5 R_{m}\right)} \int_{B\left(x_{1}, 20 R_{m}\right) \cap[0,1]^{n}}|F(A)(y)| d \mathbf{H}^{2}(y) d x_{3} d x_{1} d x_{2} . \\
& \int_{\Delta\left(x, x_{1}, x_{3}\right) \cup \Delta\left(x, x_{2}, x_{3}\right)} \mid F\left(x^{2}\right)
\end{aligned}
$$

Consider the $\Delta\left(x, x_{1}, x_{3}\right)$ integral. By Lemma 6.1, this portion of (73) is bounded by

$$
C R_{m}^{2 n} \int_{B\left(x, C R_{m}\right)}|x-y|^{2-n} F(A)(y) d y
$$

which is acceptable. The contribution of $\Delta\left(x, x_{2}, x_{3}\right)$ is similar. This completes the proof of (70). 
From (70), 68), and (59) we have in particular that

$$
\int_{\Omega_{m}} \int_{\Omega_{m}}\left|f_{x, m}\left(x_{1}\right)-f_{x, m}\left(x_{2}\right)\right| \psi_{x}\left(x_{1}\right) d x_{1} \psi_{x}\left(x_{2}\right) d x_{2} \leq C C_{1} \varepsilon\left\|\psi_{x}\right\|_{L^{1}\left(\Omega_{m}\right)}^{2} \log D .
$$

We now define the gauge $\sigma_{m+1}$ on $\Omega_{m+1}$ by the formula

$$
\sigma_{m+1}(x):=\left[f_{x, m}\right]_{\Omega_{m}, \psi_{x}}^{G}=\pi\left(\left[f_{x, m}\right]_{\Omega_{m}, \psi_{x}}\right) .
$$

From (74) and Lemma 5.3 we see that $\sigma_{m+1}$ is well-defined on $\Omega_{m+1}$. Now we prove 61 .

Fix $x_{0}, x_{1} \in \Omega_{m+1}$, and suppose that $\left|x_{0}-x_{1}\right| \leq r \leq 10 R_{m+1}$. We have to prove that

$$
\begin{aligned}
& r^{-n} \int_{B\left(x_{0}, r\right) \cap[0,1]^{n}}\left|\pi\left(\left[f_{x_{0}, m}\right]_{\Omega_{m}, \psi_{x_{0}}}\right) A\left[\left[x_{0} \rightarrow x_{2} \rightarrow x_{1}\right]\right] \pi\left(\left[f_{x_{1}, m}\right]_{\Omega_{m}, \psi_{x_{1}}}\right)^{-1}-1\right| d x_{2} \\
& \quad \leq C_{1} r\left(T_{m+1}\left(x_{0}\right)+T_{m+1}\left(x_{1}\right)\right) .
\end{aligned}
$$

By (55), we can bound the left-hand side by

$$
C r^{-n} \int_{B\left(x_{0}, r\right) \cap[0,1]^{n}}\left|\left[f_{x_{0}, m}\right]_{\Omega_{m}, \psi_{x_{0}}} A\left[\left[x_{0} \rightarrow x_{2} \rightarrow x_{1}\right]\right]-\left[f_{x_{1}, m}\right]_{\Omega_{m}, \psi_{x_{1}, m}}\right| d x_{2} .
$$

By the triangle inequality it thus suffices to prove

$$
\begin{gathered}
\mathrm{Cr}^{-n} \int_{B\left(x_{0}, r\right) \cap[0,1]^{n}}\left|\left[f_{x_{1}, m}\right]_{\Omega_{m}, \psi_{x_{0}, m}}-\left[f_{x_{1}, m}\right]_{\Omega_{m}, \psi_{x_{1}, m}}\right| d x_{2} \\
\leq \frac{1}{2} C_{1} r\left(T_{m+1}\left(x_{0}\right)+T_{m+1}\left(x_{1}\right)\right)
\end{gathered}
$$

and

$$
\begin{gathered}
C r^{-n} \int_{B\left(x_{0}, r\right) \cap[0,1]^{n}}\left|\left[f_{x_{0}, m}\right]_{\Omega_{m}, \psi_{x_{0}}} A\left[\left[x_{0} \rightarrow x_{2} \rightarrow x_{1}\right]\right]-\left[f_{x_{1}, m}\right]_{\Omega_{m}, \psi_{x_{0}, m}}\right| d x_{2} \\
\leq \frac{1}{2} C_{1} r\left(T_{m+1}\left(x_{0}\right)+T_{m+1}\left(x_{1}\right)\right) .
\end{gathered}
$$

Let us first consider (75). We can integrate out the $x_{2}$ variable to bound the left-hand side by

which we expand as

$$
C\left|\left[f_{x_{1}, m}\right]_{\Omega_{m}, \psi_{x_{0}, m}}-\left[f_{x_{1}, m}\right]_{\Omega_{m}, \psi_{x_{1}, m}}\right|,
$$

$$
C\left|\int_{\Omega_{m}} f_{x_{1}, m}(x) \varphi(x) d x\right|
$$

where $\varphi$ is the function

$$
\varphi(x):=\frac{\psi_{x_{0}, m}(x)}{\int_{\Omega_{m}} \psi_{x_{0}, m}}-\frac{\psi_{x_{1}, m}(x)}{\int_{\Omega_{m}} \psi_{x_{1}, m}} .
$$

From the support of $\varphi$ we may assume that $x$ is contained in the ball $B\left(x_{0}, 5 R_{m}\right)$.

Since $\varphi(x)$ has mean zero, we may rewrite (77) as

$$
C\left|\int_{\Omega_{m}}\left(f_{x_{1}, m}(x)-f_{x_{1}, m}\left(x^{\prime}\right)\right) \varphi(x) d x\right|
$$


for any $x^{\prime}$. Averaging over $x^{\prime} \in B\left(x_{0}, 5 R_{m}\right)$ and using the triangle inequality, we may thus bound (77) by

$$
C R_{m}^{-n} \int_{\Omega_{m} \cap B\left(x_{0}, 5 R_{m}\right)} \int_{\Omega_{m} \cap B\left(x_{0}, 5 R_{m}\right)}\left|f_{x_{1}, m}(x)-f_{x_{1}, m}\left(x^{\prime}\right)\right||\varphi(x)| d x d x^{\prime},
$$

which by Lemma 7.3 is bounded by

$$
C C_{1} R_{m}^{n}\|\varphi\|_{\infty} \int_{[0,1]^{n}} \min \left(1,\left(\frac{R_{m}}{\left|x_{0}-y\right|}\right)^{1-\kappa / 2}\right) \frac{|F(A)(y)|}{\left|x_{0}-y\right|^{n-2}} d y
$$

On the other hand, using the definition of $T_{m+1}\left(x_{0}\right)$ in (37) we have the bound

$$
\begin{aligned}
\int_{[0,1]^{n}} & \min \left(1,\left(\frac{R_{m}}{\left|x_{0}-y\right|}\right)^{1-\kappa / 2}\right) \frac{|F(A)(y)|}{\left|x_{0}-y\right|^{n-2}} d y \\
& \leq C D^{1-\kappa / 2} \int_{[0,1]^{n}} \min \left(1,\left(\frac{R_{m+1}}{\left|x_{0}-y\right|}\right)^{1-\kappa / 2}\right) \frac{|F(A)(y)|}{\left|x_{0}-y\right|^{n-2}} d y \\
& \leq C D^{1-\kappa / 2} R_{m+1} T_{m+1}\left(x_{0}\right) .
\end{aligned}
$$

Thus we can bound (77) by

$$
C C_{1} R_{m}^{n}\|\varphi\|_{\infty} D^{1-\kappa / 2} R_{m+1} T_{m+1}\left(x_{0}\right) .
$$

We now compute $\|\varphi\|_{\infty}$. From the mean-value theorem we have

$$
\psi_{x_{1}}(x)=\psi_{x_{0}}(x)+O\left(\frac{r}{R_{m}}\right)
$$

and hence

$$
\int_{\Omega_{m}} \psi_{x_{1}}(y) d y=\int_{\Omega_{m}} \psi_{x_{0}}(y) d y+O\left(\frac{r}{R_{m}}\right) R_{m}^{n} .
$$

Since $r \leq 10 R_{m+1} \ll R_{m}$, we thus see from (68) that

$$
\|\varphi\|_{\infty} \leq C \frac{r}{R_{m}} R_{m}^{-n}=\frac{C}{D} \frac{r}{R_{m+1}} R_{m}^{-n}
$$

Thus we can bound (77) by

$$
C C_{1} r D^{-\kappa / 2} T_{m+1\left(x_{0}\right)},
$$

which will be acceptable if $D$ is large enough.

Now we consider (76). By the triangle inequality and (68), we may bound the left-hand side by

$\frac{C}{r^{n} R_{m}^{n}} \int_{B\left(x_{0}, r\right) \cap[0,1]^{n}} \int_{B\left(x_{0}, 5 R_{m}\right) \cap[0,1]^{n}}\left|f_{x_{0}, m}(x) A\left[\left[x_{0} \rightarrow x_{2} \rightarrow x_{1}\right]\right]-f_{x_{1}, m}(x)\right| d x d x_{2}$.

By (69), (51), and some algebra, this can be bounded by

$$
\frac{C}{r^{n} R_{m}^{n}} \int_{B\left(x_{0}, r\right) \cap[0,1]^{n}} \int_{B\left(x_{0}, 5 R_{m}\right) \cap[0,1]^{n}}\left|A\left[\left[x \rightarrow x_{0} \rightarrow x_{2} \rightarrow x_{1} \rightarrow x\right]\right]-1_{G}\right| d x d x_{2} .
$$

Lemma 5.2 shows that for almost every $x, x_{2}$, the triangles $\Delta\left(x_{0}, x_{2}, x\right)$ and $\Delta\left(x_{1}, x_{2}, x\right)$ do not intersect $S$. Thus we may use Lemma 5.1 to estimate the previous by

$$
\frac{C}{r^{n} R_{m}^{n}} \int_{B\left(x_{0}, r\right) \cap[0,1]^{n}} \int_{B\left(x_{0}, 5 R_{m}\right) \cap[0,1]^{n}} \int_{\Delta\left(x_{0}, x_{2}, x\right) \cup \Delta\left(x_{1}, x_{2}, x\right)}|F(A)(y)| d \mathbf{H}^{2}(y) d x d x_{2}
$$


(compare with (67)). Consider the $\Delta\left(x_{0}, x_{2}, x\right)$ integral. By Lemma 6.1 (using the crude bound $|x-y|^{2-n} \leq|x-y|^{1-n} r$ when $|x-y| \leq r$ ) we can bound this portion of (178) by

$$
\operatorname{Cr} \int_{B\left(x, C R_{m}\right)}\left|x_{0}-y\right|^{n-1}|F(A)(y)| d y .
$$

By (37) we can bound this by $C(D) r T_{m+1}\left(x_{0}\right)$, which is acceptable if $C_{1}$ is large enough depending on $D$. The contribution of $\Delta\left(x_{1}, x_{2}, x\right)$ is similar but uses $T_{m+1}\left(x_{1}\right)$ instead of $T_{m+1}\left(x_{0}\right)$. This proves (75) and closes the inductive step. The proof of Proposition 4.3 is now complete.

\section{Proof of Proposition 4.4}

In this section we use Proposition 4.3 to prove Proposition 4.4

Fix $A, S, m$. From Proposition 4.3 we can find a gauge $\sigma_{m}$ on $\Omega_{m}$ obeying the bounds

$$
\left|\sigma_{m}(A)(x)\right| \leq C(D) \int_{[0,1]^{n}}\left(1+\frac{|y-x|}{R_{m}}\right)^{\kappa / 2} \frac{|F(A)(y)|}{|x-y|^{n-1}} d y
$$

for all $x \in \Omega_{m}$.

The gauge $\sigma_{m}(A)$ is currently only defined in $\Omega_{m}$. The idea is now to truncate the gauge $\sigma_{m}(A)$ away from the set (38) to make it defined everywhere; then we use a mollification argument to make the truncated gauge smooth.

We first need a Vitali covering argument. For every $x \in[0,1]^{n} \backslash \Omega_{m}$, we see from (32) that there exists a radius $0<r(x) \leq \operatorname{diam}\left([0,1]^{n}\right)$ such that

$$
r(x)^{-n / 2+1+\kappa}\left(\int_{B(x, r(x))}|F(A)(y)|^{2} d y\right)^{1 / 2} \geq \frac{1}{2} \varepsilon D^{(1-\kappa) m},
$$

and furthermore that

$$
\begin{aligned}
& r^{-n / 2+1+\kappa}\left(\int_{B(x, r)}|F(A)(y)|^{2} d y\right)^{1 / 2} \\
& \quad \leq 2 r(x)^{-n / 2+1+\kappa}\left(\int_{B(x, r(x))}|F(A)(y)|^{2} d y\right)^{1 / 2}
\end{aligned}
$$

for all $0<r \leq \operatorname{diam}\left([0,1]^{n}\right)$. From (27) and (80) we see that

$$
r(x) \leq C D^{-m}
$$

for all $x \in[0,1]^{n} \backslash \Omega_{m}$.

Since $[0,1]^{n} \backslash \Omega_{m}$ is compact, one can cover this space with only a finite number of balls $B(x, r(x))$. By the Vitali covering lemma, there thus exists a finite collection $x_{1}, \ldots, x_{N}$ of points in $[0,1]^{n} \backslash \Omega_{m}$ such that the balls $B\left(x_{j}, r\left(x_{j}\right)\right)$ are disjoint, and that the balls $B\left(x_{j}, 5 r\left(x_{j}\right)\right)$ cover $[0,1]^{n} \backslash \Omega_{m}$. In particular, these balls also cover $S$.

For each $j=1, \ldots, N$, let $\psi_{j}$ be a bump function adapted to $B\left(x_{j}, 10 r\left(x_{j}\right)\right)$ which equals one on $B\left(x_{j}, 5 r\left(x_{j}\right)\right)$, and define the function $\psi$ by

$$
\psi(x):=\sup _{j=1, \ldots, N} \psi_{j}(x) .
$$

Thus $\psi$ is a Lipschitz, piecewise smooth function which equals 1 on an open neighbourhood of $[0,1]^{n} \backslash \Omega_{m}$. Since $r(x) \leq C D^{-m} \ll R_{m}$, we see from (34) that $\psi$ vanishes on the set $\left\{x \in[0,1]^{n}: \rho(x) \geq 20 R_{m}\right\}$. (Here of course we are using 
the fact that the connection $A$ is assumed to obey the second property (28) of Proposition 4.1, which is used to prove (34).)

We define the preliminary gauge $\tilde{A}_{m}$ on $[0,1]^{n}$ by the formula

$$
\tilde{A}_{m}:=(1-\psi) \sigma_{m}(A) ;
$$

note that even though $\sigma_{m}(A)$ is only defined on $\Omega_{m}, \tilde{A}_{m}$ is defined on all of $[0,1]^{n}$ since $1-\psi$ vanishes on a neighbourhood of $[0,1]^{n} \backslash \Omega_{m}$. Also, $\tilde{A}_{m}$ is clearly gauge equivalent to $A$ on the set $\left\{x \in[0,1]^{n}: \rho(x) \geq 20 R_{m}\right\}$, and in particular is smooth on this region. Note also that $\tilde{A}_{m}$ vanishes on a neighbourhood of $S$.

Lemma 8.1 ( $\tilde{A}_{m}$ has small curvature). We have the curvature estimate

$$
\left\|F\left(\tilde{A}_{m}\right)\right\|_{M_{2}^{n / 2}\left([0,1]^{n}\right)} \leq C(D) \varepsilon .
$$

Proof. We first observe from (11), (2) and the product rule that we have the pointwise estimate

$$
\left|F\left(\tilde{A}_{m}\right)\right| \leq C|F(A)|+|\nabla \psi|\left|\sigma_{m}(A)\right| .
$$

Since $\nabla \psi$ is supported on $\Omega_{m}$ and obeys the pointwise bound $|\nabla \psi| \leq \sup _{j=1}^{N}\left|\nabla \psi_{j}\right|$, it thus suffices to show that

$$
\left\|\operatorname { s u p } _ { j } \left|\nabla \psi_{j}\left\|\sigma_{m}(A) \mid\right\|_{M_{2}^{n / 2}(\Omega)} \leq C(D) \varepsilon\right.\right.
$$

or equivalently that

$$
\int_{B(x, r) \cap \Omega_{m}} \sup _{j}\left|\nabla \psi_{j}\left(x^{\prime}\right)\right|^{2}\left|\sigma_{m}(A)\left(x^{\prime}\right)\right|^{2} d x^{\prime} \leq C(D) \varepsilon^{2} r^{n-4}
$$

for all balls $B(x, r)$.

Fix $x, r$. We may replace $\sup _{j}$ by $\sum_{j}$. Since $\nabla \psi_{j}$ is supported on $B\left(x_{j}, 10 r\left(x_{j}\right)\right)$ and has magnitude $O\left(1 / r\left(x_{j}\right)\right)$, we can bound the left-hand side of (86) by

$$
C \sum_{j} r\left(x_{j}\right)^{-2} \int_{B(x, r) \cap B\left(x_{j}, 10 r\left(x_{j}\right)\right) \cap \Omega_{m}}\left|\sigma_{m}(A)\left(x^{\prime}\right)\right|^{2} d x^{\prime} ;
$$

applying (79), we are thus reduced to showing that

$$
\begin{aligned}
& \sum_{j} r\left(x_{j}\right)^{-2} \int_{B(x, r) \cap B\left(x_{j}, 10 r\left(x_{j}\right)\right) \cap \Omega_{m}}\left(\int_{[0,1]^{n}}\left(1+\frac{\left|y-x^{\prime}\right|}{R_{m}}\right)^{\kappa / 2} \frac{|F(A)(y)|}{\left|x^{\prime}-y\right|^{n-1}} d y\right)^{2} d x^{\prime} \\
& \quad \leq C(D) \varepsilon^{2} r^{n-4} .
\end{aligned}
$$

We now split into two cases, depending on whether $r\left(x_{j}\right) \geq r$ or $r\left(x_{j}\right)<r$. First consider the terms where $r\left(x_{j}\right) \geq r$. From (82) this case can only occur when $r \leq C R_{m}$. Now for each $k \geq 0$, there are at most $O(1)$ balls $B\left(x_{j}, 10 r\left(x_{j}\right)\right)$ with $r\left(x_{j}\right) \sim 2^{k} r$ which intersect $B(x, r)$, since the balls $B\left(x_{j}, r\left(x_{j}\right)\right)$ are disjoint. Thus we can sum the series $r\left(x_{j}\right)^{-2}$ and estimate this contribution to the left-hand side of (87) by

$$
C r^{-2} \int_{B(x, r)}\left(\int_{[0,1]^{n}}\left(1+\frac{\left|y-x^{\prime}\right|}{R_{m}}\right)^{\kappa / 2} \frac{|F(A)(y)|}{\left|x^{\prime}-y\right|^{n-1}} d y\right)^{2} d x^{\prime}
$$


From (29) and a dyadic decomposition we have

$$
\int_{[0,1]^{n}:\left|x^{\prime}-y\right| \geq r}\left(1+\frac{\left|y-x^{\prime}\right|}{R_{m}}\right)^{\kappa / 2} \frac{|F(A)(y)|}{\left|x^{\prime}-y\right|^{n-1}} d y \leq C \varepsilon / r
$$

thus we may bound the previous by

$$
C \varepsilon^{2} r^{n-4}+C r^{-2} \int_{B(x, r)}\left(\int_{B\left(x^{\prime}, r\right)} \frac{|F(A)(y)|}{\left|x^{\prime}-y\right|^{n-1}} d y\right)^{2} d x^{\prime} .
$$

The variable $y$ is now restricted to the ball $B(x, 2 r)$. Since the kernel $\frac{1}{|x|^{n-1}}$ has an $L^{1}$ norm of $O(r)$ on $B(0, r)$, we can use Young's inequality to estimate the previous by

$$
C \varepsilon^{2} r^{n-4}+C\left(\int_{B(x, 2 r)}|F(A)(y)|^{2} d y\right)
$$

But this is acceptable by (27).

Now we check the contribution to 87) of the case where $r\left(x_{j}\right)<r$, which forces $B\left(x_{j}, r\left(x_{j}\right)\right) \subseteq B(x, 20 r)$. We split the $y$ integration into $\left|y-x_{j}\right| \leq 20 r\left(x_{j}\right)$ and $\left|y-x_{j}\right|>20 r\left(x_{j}\right)$. By (82), the contribution when $\left|y-x_{j}\right| \leq 20 r\left(x_{j}\right)$ is bounded by

$$
C \sum_{j: B\left(x_{j}, r\left(x_{j}\right)\right) \subseteq B(x, 20 r)} r\left(x_{j}\right)^{-2} \int_{B\left(x_{j}, 10 r\left(x_{j}\right)\right)}\left(\int_{B\left(x^{\prime}, 30 r\left(x_{j}\right)\right)} \frac{|F(A)(y)|}{\left|x^{\prime}-y\right|^{n-1}} d y\right)^{2} d x^{\prime} .
$$

Applying Young's inequality as before, we bound this by

$$
C \sum_{j: B\left(x_{j}, r\left(x_{j}\right)\right) \subseteq B(x, 20 r)} \int_{B\left(x_{j}, 40 r\left(x_{j}\right)\right)}|F(A)(y)|^{2} d y .
$$

Applying (81), we can bound this by

$$
C \sum_{j: B\left(x_{j}, r\left(x_{j}\right)\right) \subseteq B(x, 20 r)} \int_{B\left(x_{j}, r\left(x_{j}\right)\right)}|F(A)(y)|^{2} d y .
$$

Since the balls $B\left(x_{j}, r\left(x_{j}\right)\right)$ are disjoint, this is bounded by

$$
C \int_{B(x, 20 r)}|F(A)(y)|^{2} d y
$$

and the claim follows from (27).

Now we consider the contribution when $\left|y-x_{j}\right|>20 r\left(x_{j}\right)$. In this case the $x^{\prime}$ variable is essentially irrelevant, and we can estimate this contribution by

$$
C \sum_{j: B\left(x_{j}, r\left(x_{j}\right)\right) \subseteq B(x, 20 r)} r\left(x_{j}\right)^{n-2}\left(\int_{\left|y-x_{j}\right|>20 r\left(x_{j}\right)}\left(1+\frac{\left|y-x_{j}\right|}{R_{m}}\right)^{\kappa / 2} \frac{|F(A)(y)|}{\left|x_{j}-y\right|^{n-1}} d y\right)^{2} ;
$$

from (82) we may crudely bound this by

$$
C \sum_{j: B\left(x_{j}, r\left(x_{j}\right) \subseteq \subseteq B(x, 20 r)\right.} r\left(x_{j}\right)^{n-2}\left(\int_{\left|y-x_{j}\right|>20 r\left(x_{j}\right)}\left(\frac{\left|y-x_{j}\right|}{r\left(x_{j}\right)}\right)^{\kappa / 2} \frac{|F(A)(y)|}{\left|x_{j}-y\right|^{n-1}} d y\right)^{2} .
$$

From (81) and Hölder's inequality we have

$$
\int_{\left|y-x_{j}\right| \leq r}|F(A)(y)| \leq C r^{n-1-\kappa} r\left(x_{j}\right)^{-n / 2+1+\kappa}\left(\int_{B\left(x_{j}, r\left(x_{j}\right)\right)}|F(A)(y)|^{2} d y\right)^{1 / 2}
$$


for any $0<r \leq \operatorname{diam}\left([0,1]^{n}\right)$. From dyadic decomposition we thus have

$$
\begin{aligned}
\int_{\left|y-x_{j}\right|>20 r\left(x_{j}\right)} & \left(1+\frac{\left|y-x_{j}\right|}{R_{m}}\right)^{\kappa / 2} \frac{|F(A)(y)|}{\left|x_{j}-y\right|^{n-1}} d y \\
\leq & \sum_{k \geq 0} C 2^{k \kappa / 2}\left(2^{k} r\left(x_{j}\right)\right)^{-(n-1)}\left(2^{k} r\left(x_{j}\right)\right)^{n-1-\kappa} \\
& r\left(x_{j}\right)^{-n / 2+1+\kappa}\left(\int_{B\left(x_{j}, r\left(x_{j}\right)\right)}|F(A)(y)|^{2} d y\right)^{1 / 2} \\
\leq & C r\left(x_{j}\right)^{-n / 2+1}\left(\int_{B\left(x_{j}, r\left(x_{j}\right)\right)}|F(A)(y)|^{2} d y\right)^{1 / 2}
\end{aligned}
$$

since the $k$ summation is convergent. In particular, we have

$$
(89) \leq C\left(\int_{B\left(x_{j}, r\left(x_{j}\right)\right)}|F(A)(y)|^{2} d y\right)^{1 / 2} .
$$

Thus we can bound this contribution by (88), which is acceptable as before. This proves (84).

We are almost done, except that $\sigma_{m}$, and hence the $\tilde{A}_{m}$, is not necessarily smooth. Fortunately this can be easily resolved by regularizing $\sigma_{m}$.

Fix $m$. Since $\sigma_{m}$ is locally Lipschitz on $\Omega_{m}$, it lies in the Sobolev space $W^{1, p}$ on the support of $1-\psi$ for any $n<p<\infty$. In particular, we can create a sequence $\sigma_{m, j}$ of smooth gauges which converge strongly in $W^{1, p}$ to $\sigma_{m}$ as $j \rightarrow \infty$ on the support of $1-\psi$; note that $W^{1, p}$ functions are Hölder continuous and so there is no difficulty keeping $\sigma_{m, j}$ on the Lie group $G$.

Define the gauges $\tilde{A}_{m, j}$ by

$$
\tilde{A}_{m, j}=(1-\psi) \sigma_{m, j}(A) .
$$

Then by construction $\tilde{A}_{m, j}$ is smooth and vanishes near $S$. Now we compare the curvatures of $\tilde{A}_{m, j}$ and $\tilde{A}_{m}$. First we use (11) to rewrite

$$
\tilde{A}_{m, j}=(1-\psi) \tilde{\sigma}_{m, j}\left(\sigma_{m}(A)\right)
$$

where $\tilde{\sigma}_{m, j}:=\sigma_{m, j} \sigma_{m}^{-1}$. From (1) and the product rule we have

$$
F\left(\tilde{A}_{m, j}\right)=(1-\psi) F\left(\tilde{\sigma}_{m, j}\left(\sigma_{m} A\right)\right)-\psi(1-\psi) \tilde{\sigma}_{m, j}\left(\sigma_{m}(A)\right) \wedge \tilde{\sigma}_{m, j}\left(\sigma_{m}(A)\right)
$$

and similarly

$$
F\left(\tilde{A}_{m}\right)=(1-\psi) F\left(\sigma_{m}(A)\right)-\psi(1-\psi) \sigma_{m}(A) \wedge \sigma_{m}(A) .
$$

On the other hand, from (9) we have

$$
F\left(\tilde{\sigma}_{m, j}\left(\sigma_{m}(A)\right)\right)=\tilde{\sigma}_{m, j}^{-1} F\left(\sigma_{m}(A)\right) \tilde{\sigma}_{m, j}
$$

and hence

$$
\begin{aligned}
& \quad F\left(\tilde{A}_{m, j}\right)-\tilde{\sigma}_{m, j}^{-1} F\left(\sigma_{m}(A)\right) \tilde{\sigma}_{m, j} \\
& \quad=\psi(1-\psi)\left(\tilde{\sigma}_{m, j}\left(\sigma_{m}(A)\right) \wedge \sigma_{m, j}\left(\sigma_{m}(A)\right)-\tilde{\sigma}_{m, j}^{-1}\left(\sigma_{m}(A) \wedge \sigma_{m}(A)\right) \tilde{\sigma}_{m, j} .\right.
\end{aligned}
$$

Since $\tilde{\sigma}_{m, j}$ converges to the identity in $W^{1, p}$, and $\sigma_{m}(A)$ is bounded on the support of $1-\psi$, we see that the right-hand side converges to zero in $L^{p}$, and hence in $M_{2}^{n / 2}$. From (84) we thus see that $\left\|F\left(\tilde{A}_{m, j}\right)\right\|_{M_{2}^{n / 2}\left([0,1]^{n}\right)} \leq \varepsilon$ if $j$ is sufficiently 
small. The claim then follows by setting $A:=\tilde{A}_{m, j}$ for this value of $j$. The proof of Proposition 4.4 is now complete.

\section{Proof of Theorem 4.6}

We now prove Theorem 4.6. Our arguments are essentially those in $[6$, but with Lebesgue spaces replaced by their Morrey counterparts. A similar argument has appeared in [1].

Let $K \gg 1$ be an absolute constant to be chosen later, and let $0<\varepsilon \ll 1$ be sufficiently small depending on $K$. Define $\mathbf{U}_{\varepsilon}^{*}$ to denote the space of connections $A \in \mathbf{U}_{\varepsilon}$ which are gauge equivalent (via a smooth gauge) to a smooth Coulomb gauge $A_{\text {coulomb }}$ such that

$$
\left\|A_{\text {coulomb }}\right\|_{M_{2,1}^{n / 2}\left([0,1]^{n}\right)} \leq K \varepsilon .
$$

Clearly $\mathbf{U}_{\varepsilon}^{*}$ is invariant under gauge transformations.

The main estimate is already contained in

Lemma 9.1 (Bootstrap estimate). For any $A \in \boldsymbol{U}_{\varepsilon}^{*}$, we can bootstrap (90) to

$$
\left\|A_{\text {coulomb }}\right\|_{M_{2,1}^{n / 2}\left([0,1]^{n}\right)} \leq K \varepsilon / 2 .
$$

Also, we have (40).

Proof. From elliptic estimates (Proposition 3.5) we have

$$
\left\|A_{\text {coulomb }}\right\|_{M_{2,1}^{n / 2}\left([0,1]^{n}\right)} \leq C\left\|d A_{\text {coulomb }}\right\|_{M_{2}^{n / 2}\left([0,1]^{n}\right)^{n}} .
$$

By (11), (16), (12), we thus have

$$
\left\|A_{\text {coulomb }}\right\|_{M_{2,1}^{n / 2}\left([0,1]^{n}\right)} \leq C\left(\|F(A)\|_{M_{2}^{n / 2}\left([0,1]^{n}\right)}+\left\|A_{\text {coulomb }}\right\|_{M_{2,1}^{n / 2}\left([0,1]^{n}\right)}^{2}\right) .
$$

The claims then follow from (90), (39) if $K$ is sufficiently large and $\varepsilon$ sufficiently small depending on $K$.

Fix $A \in \mathbf{U}_{\varepsilon}$. To prove Theorem 1.6, it suffices from the above lemma to show that $A \in \mathbf{U}_{\varepsilon}^{*}$.

We exploit the smoothness of $A$ by choosing an exponent $n / 2<p<n$ (e.g., $p=3 n / 4$ will do). We introduce the one-parameter family of connections $A_{t}$ for $t \in[0,1]$ by

$$
A_{t}(x):=t A(t x) .
$$

One may easily verify that the $A_{t}$ lie in $\mathbf{U}_{\varepsilon}$, and that the map $t \mapsto A_{t}$ is continuous in the $M_{2,1}^{p}$ topology. Also, $A_{0}=0$ is clearly in $\mathbf{U}_{\varepsilon}^{*}$. In order to show that $A_{1}$ is in $\mathbf{U}_{\varepsilon}^{*}$ it thus suffices by standard continuity arguments to prove

Proposition 9.2 (Continuity of the Coulomb gauge construction in smooth norms). Let $0<X<\infty, n / 2<p<n$, and let $A \in \boldsymbol{U}_{\varepsilon}^{*}$ be such that

$$
\|A\|_{M_{2,1}^{p}\left([0,1]^{n}\right)} \leq X .
$$

Then there exists a quantity $\delta_{X}>0$ depending only on $X, G, n, p$, $\varepsilon$, such that

$$
\left\{A+\lambda \in \boldsymbol{U}_{\varepsilon}:\|\lambda\|_{M_{2,1}^{p}\left([0,1]^{n}\right)} \leq \delta_{X}\right\} \subset \boldsymbol{U}_{\varepsilon}^{*} .
$$


Proof. Fix $p$; all our constants are allowed to depend on $p$. We use $C_{X}$ to denote quantities which also depend on $X$.

Step 1. Estimate the Coulomb gauge in smooth norms.

From (92), 1), 17) we have

$$
\|F(A)\|_{M_{2}^{p}\left([0,1]^{n}\right)} \leq C_{X} .
$$

From (12) we thus have

$$
\left\|F\left(A_{\text {coulomb }}\right)\right\|_{M_{2}^{p}\left([0,1]^{n}\right)} \leq C_{X} .
$$

From elliptic estimates (Proposition 3.5) and (1) we thus have

$$
\left.\left\|A_{\text {coulomb }}\right\|_{M_{2,1}^{p}\left([0,1]^{n}\right)} \leq C_{X}+C\left\|A_{\text {coulomb }} \wedge A_{\text {coulomb }}\right\|_{M_{2}^{p}\left([0,1]^{n}\right)}\right) .
$$

By (18), (90) we thus obtain

$$
\left\|A_{\text {coulomb }}\right\|_{M_{2,1}^{p}\left([0,1]^{n}\right)} \leq C_{X} .
$$

Next, let $\sigma_{\text {coulomb }}$ be the gauge transformation such that $A_{\text {coulomb }}=\sigma_{\text {coulomb }}(A)$. From (2) we have

$$
d \sigma_{\text {coulomb }}=\sigma_{\text {coulomb }} A-A_{\text {coulomb }} \sigma_{\text {coulomb }} .
$$

Differentiating this, we have the pointwise estimate

$$
\left|\nabla^{2} \sigma_{\text {coulomb }}\right| \leq C\left(\left|\sigma_{\text {coulomb }}\right|\left(|\nabla A|+\left|\nabla A_{\text {coulomb }}\right|\right)+\left|d \sigma_{\text {coulomb }}\right|\left(|A|+\left|A_{\text {coulomb }}\right|\right)\right) \text {. }
$$

We substitute (94) into this estimate. Since $G$ is compact, $\sigma_{\text {coulomb }}$ is bounded, and we thus obtain the pointwise bounds

$$
\left|\nabla^{2} \sigma_{\text {coulomb }}\right| \leq C\left(1+|\nabla A|+|A|^{2}+\left|\nabla A_{\text {coulomb }}\right|+\left|A_{\text {coulomb }}\right|^{2}\right) .
$$

From (94) and the boundedness of $\sigma_{\text {coulomb }}$ we in fact have

$$
\begin{aligned}
& \left|\sigma_{\text {coulomb }}\right|+\left|\nabla \sigma_{\text {coulomb }}\right|+\left|\nabla^{2} \sigma_{\text {coulomb }}\right| \\
& \quad \leq C\left(1+|\nabla A|+|A|^{2}+\left|\nabla A_{\text {coulomb }}\right|+\left|A_{\text {coulomb }}\right|^{2}\right) .
\end{aligned}
$$

From (92), (93), and (17) we thus have

$$
\left\|\sigma_{\text {coulomb }}\right\|_{M_{2,2}^{p}\left([0,1]^{n}\right)} \leq C_{X} .
$$

\section{Step 2. Pass to the Coulomb gauge.}

From (2), (95), (17), we see that the gauge transformation $\tilde{A} \mapsto \sigma_{\text {coulomb }}(\tilde{A})$ is uniformly continuous in a small neighbourhood of $A$ (in the $M_{2,1}^{p}$ topology). Since $\mathbf{U}_{\varepsilon}^{*}$ and $\mathbf{U}_{\varepsilon}$ are invariant under gauge transformations, we thus see that to prove Proposition 9.2 it suffices to do so in the case $A=A_{\text {coulomb }}$.

Step 3. Apply perturbation theory to the Coulomb gauge.

Fix $\lambda$ as in the proposition. In order to show that $A+\lambda \in \mathbf{U}_{\varepsilon}^{*}$, we first need to construct a gauge $\sigma$ such that

$$
\begin{aligned}
d_{*}(\sigma(A+\lambda)) & =0 \text { on }[0,1]^{n}, \\
n \cdot(\sigma(A+\lambda)) & =0 \text { on } \partial[0,1]^{n} .
\end{aligned}
$$

We use a perturbative argument. Write $\sigma=\exp (U)$. By (2) the above non-linear elliptic problem can then be written as

$$
\begin{gathered}
\Delta U=d_{*} F(U, A+\lambda) \text { on } \Omega, \\
n_{\alpha} d^{\alpha} U=F(U, A+\lambda) \text { on } \partial \Omega,
\end{gathered}
$$


where

$$
F(U, A+\lambda):=d U-d \exp (U) \cdot \exp (-U)+\exp (U) \cdot(A+\lambda) \cdot \exp (-U) .
$$

We therefore set up the iteration scheme

$$
\begin{gathered}
\Delta U^{(j+1)}=d_{*} F\left(U^{(j)}, A+\lambda\right) \text { on } \Omega, \\
n_{\alpha} d^{\alpha} U^{(j+1)}=F\left(U^{(j)}, A+\lambda\right) \text { on } \partial \Omega, \\
\int U^{(j+1)}=0,
\end{gathered}
$$

where $U^{0}:=0$. Note that $U^{(j+1)}$ is uniquely defined by the classical theory of the Neumann problem; indeed, $U^{(j+1)}$ can be defined in terms of the Hodge decomposition of $F\left(U^{(j)}, A+\lambda\right)$.

We derive some bounds on $U^{(j+1)}$. From the boundary conditions on $A$ we have $F\left(U^{(j)}, \lambda\right)=F\left(U^{(j)}, A+\lambda\right)$ on $\partial[0,1]^{n}$. From Neumann problem regularity (Proposition 3.6) we thus have

$$
\left\|U^{(j+1)}\right\|_{M_{2,2}^{p}\left([0,1]^{n}\right)} \leq C\left\|d_{*} F\left(U^{(j)}, A+\lambda\right)\right\|_{M_{2}^{p}\left([0,1]^{n}\right)}+C\left\|F\left(U^{(j)}, \lambda\right)\right\|_{M_{2,1}^{p}\left([0,1]^{n}\right)} .
$$

From the chain rule and Definition 4.5 we observe the pointwise bounds

$\left|d_{*} F\left(U^{(j)}, A+\lambda\right)\right| \leq C\left(\left|U^{(j)}\right|\left|\nabla^{2} U^{(j)}\right|+\left|\nabla U^{(j)}\right|^{2}+|A|\left|\nabla U^{(j)}\right|+|\lambda|\left|\nabla U^{(j)}\right|+|\nabla \lambda|\right)$.

By (17), (40), (18) we thus have

$$
\begin{aligned}
& \left\|d_{*} F\left(U^{(j)}, A+\lambda\right)\right\|_{M_{2}^{p}\left([0,1]^{n}\right)} \\
& \quad \leq C\left(\left\|U^{(j)}\right\|_{M_{2,2}^{p}\left([0,1]^{n}\right)}^{2}+K \varepsilon\left\|U^{(j)}\right\|_{M_{2,2}^{p}\left([0,1]^{n}\right)}+\|\lambda\|_{M_{2,1}^{p}\left([0,1]^{n}\right)}\right) .
\end{aligned}
$$

Similarly we have

$$
\left\|F\left(U^{(j)}, \lambda\right)\right\|_{M_{2,1}^{p}\left([0,1]^{n}\right)} \leq C\left(\left\|U^{(j)}\right\|_{M_{2,2}^{p}\left([0,1]^{n}\right)}^{2}+\|\lambda\|_{M_{2,1}^{p}}\left([0,1]^{n}\right)\right) .
$$

Thus we have

$\left\|U^{(j+1)}\right\|_{M_{2,2}^{p}\left([0,1]^{n}\right)} \leq C\left(\left\|U^{(j)}\right\|_{M_{2,2}^{p}\left([0,1]^{n}\right)}^{2}+K \varepsilon\left\|U^{(j)}\right\|_{M_{2,2}^{p}\left([0,1]^{n}\right)}+\|\lambda\|_{M_{2,1}^{p}}\left([0,1]^{n}\right)\right)$.

If $\delta_{X}$ is sufficiently small, we thus obtain inductively

$$
\left\|U^{(j)}\right\|_{M_{2,2}^{p}\left([0,1]^{n}\right)} \leq C \delta_{X} .
$$

Adapting this scheme to differences, we thus see that $U^{(j)}$ converges in $M_{2,2}^{p}\left([0,1]^{n}\right)$ to a solution $U$ with

$$
\|U\|_{M_{2,2}^{p}\left([0,1]^{n}\right)} \leq C \delta_{X} .
$$

In particular, from Corollary 14 we see that $U$ has some Hölder continuity. Standard elliptic regularity theory can then be used to bootstrap this regularity, eventually concluding that $U$ is smooth. Exponentiating this (using (17)) we thus obtain a smooth Coulomb gauge $\sigma(A+\lambda)$ with

$$
\|\sigma-1\|_{M_{2,2}^{p}\left([0,1]^{n}\right)},\left\|\sigma^{-1}-1\right\|_{M_{2,2}^{p}\left([0,1]^{n}\right)} \leq C \delta_{X},
$$

where 1 is the identity element of $G$. From (2), (17), (92) we thus have

$$
\|\sigma(A+\lambda)-A\|_{M_{2,1}^{p}\left([0,1]^{n}\right)} \leq C_{X} \delta_{X}
$$

and hence

$$
\|\sigma(A+\lambda)-A\|_{M_{2,1}^{n / 2}\left([0,1]^{n}\right)} \leq C_{X} \delta_{X}
$$


If $\delta_{X}$ is sufficiently small depending on $X$, we thus see from (91) that

$$
\|\sigma(A+\lambda)\|_{M_{2,1}^{n / 2}\left([0,1]^{n}\right)} \leq K \varepsilon
$$

as desired.

The proof of Theorem 4.6 is now complete. This completes all the steps necessary to prove Theorem 1.1

\section{REFERENCES}

[1] Y. Meyer, T. Rivière, Partial regularity results for a class of stationary Yang-Mills fields in high dimension, Rev. Mat. Iberoamericana, 19 (2003), 195-219.

[2] J. Price, A monotonicity formula for Yang-Mills fields, Manuscripta Math. 43 (1983), no. 2-3, 131-166. MR 84m:58033

[3] E. M. Stein, Harmonic Analysis, Princeton University Press, 1993. MR 95c:42002

[4] M. Taylor, Partial Differential Equations III, Springer-Verlag, New York, 1997. MR 98k:35001

[5] G. Tian, Gauge theory and calibrated geometry, I, Annals of Math. 151 (2000), 193-268. MR 2000m:53074

[6] K. Uhlenbeck, Connections with $L^{p}$ bounds on curvature, Commun. Math. Phys. 83 (1982), 31-42. MR 83e:53035

[7] K. Uhlenbeck, Removing singularities in Yang-Mills fields, Commun. Math. Phys. 83 (1982), 11-29. MR 83e:53034

Department of Mathematics, University of California at los Angeles, Los Angeles, CALifornia 90095-1555

E-mail address: tao@math.ucla.edu

Department of Mathematics, Massachusetts institute of Technology, Cambridge, Massachusetts 02139

E-mail address: tian@math.mit.edu 